\title{
DECOMPOSITIONS FOR RELATIVELY NORMAL LATTICES
}

\author{
JAMES B. HART AND CONSTANTINE TSINAKIS
}

\begin{abstract}
Continuing the work begun in Snodgrass and Tsinakis [26, 27], we develop a family of decomposition theorems for classes of relatively normal lattices. These results subsume and are inspired by known decomposition theorems in order-algebra due to P. Conrad and D. B. McAlister. As direct corollaries of the main results, we obtain corresponding decomposition theorems for classes of partially ordered sets.
\end{abstract}

\section{INTRODUCTION}

A pcset $\boldsymbol{P}=(P ; \leq)$ is a root-system provided the principal upper set $\uparrow p=$ $\{x \in P: p \leq x\}$ is a chain for all $p \in P$. A maximal chain in $\mathbf{P}$ is called a root. A lower-bounded, distributive lattice is relatively normal provided its set of prime ideals is a root-system under set-inclusion.

The class of relatively normal lattices is defined by a universal-existential, positive sentence (see Theorem 1.1(4)); in all that follows, we shall denote this class by $\mathscr{R} \mathscr{N}$.

The terms "normal" and "relatively normal" were coined by Monteiro in connection with his studies on filters and topological spaces (Monteiro [24, 25]). A bounded, distributive lattice is said to be normal if each of its prime ideals is contained in a unique maximal ideal. Monteiro proved that a topological space is normal (in the usual topological sense, with no separation axioms assumed) if and only if its lattice of open sets is normal. As the term suggests, a lower-bounded, distributive lattice is relatively normal if and only if each of its closed subintervals is a normal lattice. Monteiro showed that a similar topological connection exists for relatively normal lattices: a topological space is hereditarily normal (i.e., every subspace is normal) if and only if its open sets form a relatively normal lattice.

The class $\mathscr{R} \mathscr{N}$ properly includes the class of dual relative Stone lattices. For example, Mandelker [20] has shown that the lattice of cozero-sets of any topological space is relatively normal; such lattices are rarely dual relative Stone lattices. In addition, a number of arithmetical varieties have the property that the compact congruences of each member form a relatively normal lattice. Among these are representable varieties of lattice-ordered groups ( $l$-groups), Riesz spaces, $f$ rings, and relative Stone algebras.

Received by the editors December 11, 1990.

1991 Mathematics Subject Classification. Primary 06D05; Secondary 06D15, 06D20, 06A06, 06A23, 06F15, 06F20. 
Some relevant publications include Beazer [4], Bordalo [6], Bordalo and Priestly [7], Cornish [13, 14], Davey [16], Johnstone [19], Mandelker [20], Monteiro [24, 25], Snodgrass and Tsinakis [26, 27], and Zaanen [28]. The reader is advised that some of the cited authors express normality as a property of the lattice of closed sets of a topological space. Hence, their use of the terms "normal" and "relatively normal" refer to conditions dual to those presented here.

The paper by Snodgrass and Tsinakis [27] is concerned with the class $\mathscr{I R N}$ of all algebraic, distributive lattices whose compact elements form a relatively normal lattice. This study has yielded a large body of information for members of this class and has shown that many fundamental results for the aforementioned varieties admit a purely lattice-theoretic development. The classical definition for the ordinal sum of two posets plays a key role in these considerations: The ordinal sum of the disjoint posets $\boldsymbol{P}$ and $\boldsymbol{Q}$ is the poset $\boldsymbol{P} \oplus \boldsymbol{Q}=(P \cup Q ; \leq)$, where the individual orders of $\boldsymbol{P}$ and $\boldsymbol{Q}$ are retained and $p<q$ for all $p \in \boldsymbol{P}$ and $q \in \boldsymbol{Q}$.

Snodgrass and Tsinakis define an ordinal extension of a poset $\boldsymbol{P}$ to be the ordinal sum of $\boldsymbol{P}$ with a chain $C$. Based upon this notion, they define an ordinal extension of an element of a member of the class $\mathscr{G R N}$ (see $\S 2$ ) and proceed to show that ordinal extensions in members of this class display many striking properties first observed in the setting of $l$-groups. Their study culminates in the proof of a purely lattice-theoretic analog of Conrad's Finite Basis Theorem for l-groups:

Theorem A. For a lower-bounded, distributive lattice $\boldsymbol{D}$ the following are equivalent:

(1) D is relatively normal and every set of pairwise orthogonal elements in $D$ is finite.

(2) The ideal lattice, $\mathbf{I}(\boldsymbol{D})$, of $\boldsymbol{D}$ is isomorphic to the lattice $\mathbf{O}(\boldsymbol{R})$ of lower sets of some root-system $\boldsymbol{R}$ having only finitely many roots.

(3) There exists an ascending chain ${ }^{1} D^{0} \subset D^{1} \cdots \subset D^{m}=\mathbf{D}$ of ideals of $\boldsymbol{D}$ satisfying the following conditions:

(a) For $0 \leq k \leq m, \boldsymbol{D}^{k}$ is the join in $\mathbf{I}(\boldsymbol{D})$ of a set $\mathbf{B}(k)=\left\{\boldsymbol{D}_{j}^{k}: j \in\right.$ $\left.J_{k}\right\}$, where

(b) $\mathbf{B}(0)$ is a finite set of orthogonal ideals of $\boldsymbol{D}$ which are chains;

(c) For $0<k \leq m$, each $\mathbf{D}_{j}^{k}$ is either in $\mathbf{B}(k-1)$ or else a proper ordinal extension of the join in $\mathbf{I}(\boldsymbol{D})$ if two or more of the ideals in $\mathbf{B}(k-1)$.

The proof of this theorem relies upon two consequences of the theory for $\mathscr{I R N}$. First, the equivalence of statements (1) and (2) is established by appealing to a key result characterizing all bialgebraic (algebraic and dually algebraic) members of $\mathscr{Y R N}$ (Snodgrass and Tsinakis [26]):

A member of the class $\mathscr{I R N}$ is bialgebraic if and only if each of its compact elements has only finitely many values.

A more precise statement of this result is given as Corollary 1.4. (In a poset, $p$ is a value of $x$ provided $p$ is maximal with respect to not exceeding $x$; see $\S 1$.

\footnotetext{
${ }^{1}$ In all that follows, we use " $\subset$ " or " $\supset$ " to denote proper set inclusion.
} 


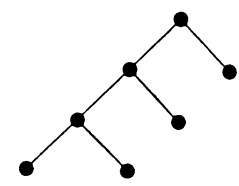

FigURE 1. Filet configuration

The second consequence lies at the heart of the decomposition presented in statement (3). The properties of proper ordinal extensions force the ideals in $\mathbf{B}(k)$ to partition the ideals in $\mathbf{B}(k-1)$. In other words, if $\mathbf{S}\left(\boldsymbol{D}_{n}^{j}\right)$ denotes the ideals in $\boldsymbol{B}(k-1)$ contained in $\boldsymbol{D}_{j}^{n}$, then the sets $\mathbf{S}\left(\boldsymbol{D}_{j}^{n}\right)$ form a partition of $\mathbf{B}(k-1)$. Moreover, this partitioning is unique, being completely determined by the structure of the lattice.

The objective of this paper is to develop the most general conditions for relatively normal lattices which guarantee the existence of decompositions similar in kind to Theorem A. The main result of this study is the following extension of Theorem A:

Theorem B. For a lower-bounded, distributive lattice $\boldsymbol{D}$ the following are equivalent:

(1) D is relatively normal and the quotient lattice $\mathrm{D} / \boldsymbol{\Theta}$, relative to the Glivenko congruence $\Theta$, satisfies the descending chain condition.

(2) The ideal lattice $\mathbf{I}(\boldsymbol{D})$ is isomorphic to the lattice $\mathbf{O}(\boldsymbol{R})$ of lower sets of some root-system $\boldsymbol{R}$ which does not contain an infinite filet configuration.

(3) There exists an ordinal $\delta>0$ and an ascending chain $\left\{D^{\beta}: \beta<\delta\right\}$ of ideals of $\boldsymbol{D}$ satisfying the following conditions:

(A) $\boldsymbol{D}=\bigcup\left\{\boldsymbol{D}^{\beta}: \beta<\delta\right\}$

(B) $D^{\beta}$ is the join $\bigvee\left\{\boldsymbol{D}_{j}^{\beta}: j \in J_{\beta}\right\}$ in $\mathbf{I}(\mathbf{D}), 0 \leq \beta<\delta$, where

(C) $\left\{\boldsymbol{D}_{j}^{0}: j \in J_{0}\right\}$ is a family of orthogonal ideals of $\mathbf{D}$ which are chains;

(D) If $\beta$ is a successor ordinal, then each $\boldsymbol{D}_{j}^{\beta}$ is either a joinand of $D^{\beta-1}$ or else a proper ordinal extension of the join of two or more joinands of $\boldsymbol{D}^{\beta-1}$;

(E) If $0<\beta$ is a limit ordinal, then each $\boldsymbol{D}_{j}^{\beta}$ is a (possibly trivial) ordinal extension of the union of a chain of joinands, one from each $D^{\xi}, 0 \leq \xi<\beta$.

The proof of this result, as well as the pertinent concepts, are given in $\S 3$. Stated simply, the Glivenko congruence identifies all elements of $\boldsymbol{D}$ whose corresponding principal ideals have the same pseudocomplement in $\mathbf{I}(\boldsymbol{D})$. A filet configuration in a poset $\boldsymbol{P}$ is a (possibly infinite) subposet of $\boldsymbol{P}$ having the Hasse diagram of Figure 1.

We may use the equivalence of statements (2) and (3) in Theorem B to obtain the following result for root-systems:

Theorem $\mathbf{C}$. For a poset $\boldsymbol{R}$ the following are equivalent:

(1) $\boldsymbol{R}$ is a root-system, and every filet configuration in $\boldsymbol{R}$ is finite.

(2) There exists an ordinal $0<\delta$ and an ascending chain $\left\{\boldsymbol{R}^{\beta}: \beta<\delta\right\}$ of lower sets of $\boldsymbol{R}$ such that

(A) $\boldsymbol{R}=\bigcup\left\{\boldsymbol{R}^{\beta}: \beta<\delta\right\}$. 
(B) $\boldsymbol{R}^{\beta}$ is the disjoint union of a set $\left\{\boldsymbol{R}_{j}^{\beta}: j \in J_{\beta}\right\}, 0 \leq \beta<\delta$, where

(C) $\left\{\boldsymbol{R}_{j}^{0}: j \in J_{0}\right\}$ is a family of pairwise disjoint chains of $\boldsymbol{R}$;

(D) If $\beta$ is a successor ordinal, then each $\boldsymbol{R}_{j}^{\beta}$ is either a component of $\boldsymbol{R}^{\beta-1}$ or else a proper ordinal extension of the disjoint union of two or more components of $\boldsymbol{R}^{\beta-1}$;

(E) If $0<\beta$ is a limit ordinal, then each $\boldsymbol{R}_{j}^{\beta}$ is a (possibly trivial) ordinal extension of the union of a chain of components, one from each $\boldsymbol{R}^{\xi}, 0 \leq \xi<\beta$.

We also address the important unanswered question of whether every relatively normal lattice is isomorphic to the lattice of all principal convex $l$ subgroups (compact congruence relations) of an l-group. An affirmative answer for finite relatively normal lattices has been established in Conrad [10]. Theorem D generalizes this result.

Theorem D. Let $\boldsymbol{D}$ be a lower-bcunded, distributive lattice such that the quotient lattice $\mathrm{D} / \boldsymbol{\Theta}$, relative to the Glivenko congruence $\boldsymbol{\Theta}$, satisfies the descending chain condition. Then $\boldsymbol{D}$ is relatively normal (and hence satisfies the conditions of Theorem $\mathbf{B}$ ) if and only if $\mathbf{D}$ is isomorphic to the lattice of all principal convex l-subgroups of an abelian l-group.

In $\S 1$, we provide a brief review of some relevant concepts from the theory of algebraic lattices and conclude the section with the characterization of bialgebraic members of the class $\mathscr{G R} \mathscr{N}$ established in Snodgrass and Tsinakis [27].

In $\S 2$, we summarize the theory of ordinal extensions developed in the same paper.

In $\S \S 3$ and 4, we draw upon and extend the results summarized in $\S \S 1$ and 2 to prove the main results of this study. Two obstacles must be overcome before we prove Theorem B. The first is to establish the equivalence of statements (1) and (2). We accomplish this in Theorem 3.3 and Corollary 3.4. Second, we must prove that ordinal decompositions exist in members of the class $\mathscr{G R} \mathscr{N}$. We accomplish this through Lemmas 4.5, 4.6, and Construction 4.7. The key to establishing Theorem $B$ lies in proving that if the ideal $D^{\beta}$ has been constructed and $D^{\beta} \neq D$, then the ideal $D^{\beta+1} \supset D^{\beta}$ may be constructed. The kernel of this argument is contained in Lemma 4.8.

In $\S 5$, we show that by placing additional restrictions on the lattice $D$, several corollaries to the main theorem can be deduced which provide considerable control over the components of the decomposition. In so doing, we obtain a new Proof of Theorem A.

The decomposition theory presented in this paper generalizes and owes a considerable debt to studies in order-algebra. In particular, Conrad's fundamental contributions have been instrumental in our considerations. In $\S 6$, in addition to proving Theorem $\mathrm{D}$, we trace the history of these earlier results and outline how they have influenced our work.

\section{Preliminaries}

In this section, we review some relevant concepts from the basic theory of algebraic lattices. In the interest of brevity, no proofs will be given. For proofs 
and notions not defined here, the reader is referred to Balbes and Dwinger [3], Crawley and Dilworth [15], Grätzer [17], or McKenzie et al. [23]. With the exception of Theorem 1.1, all results in this section concerning the classes $\mathscr{R} \mathscr{N}$ and $\mathscr{I R N}$ are taken from Snodgrass and Tsinakis [27].

An algebraic, distributive lattice $\boldsymbol{L}$ satisfies the join-infinite distributive law (JID):

$$
x \wedge \bigvee\left\{y_{i}: i \in I\right\}=\bigvee\left\{x \wedge y_{i}: i \in I\right\}
$$

Consequently, algebraic, distributive lattices are Heyting lattices; that is, for all $a, b \in \boldsymbol{L}$, the ideal $\{x \in \boldsymbol{L}: a \wedge x \leq b\}$ has a largest element. We call this element the relative pseudocomplement of $a$ with respect to $b$ and denote it by $a \rightarrow b$. We shall be especially interested in the pseudocomplements $a^{*}=a \rightarrow \perp$ and the set

$$
\mathbf{B}(\boldsymbol{L})=\left\{a \in \boldsymbol{L}: a=a^{* *}\right\}
$$

of skeletal elements of $\boldsymbol{L}$. It is well known that $(\mathbf{B}(\boldsymbol{L}) ; \wedge, \sqcup, *, \perp, T)$ is a Boolean algebra with join operation defined by $a \sqcup b=(a \vee b)^{* *}$. (See, for example, Balbes and Dwinger [3] or Grätzer [17].) We will call $\mathbf{B}(L)$ the Boolean skeleton of $\boldsymbol{L}$.

Let $\boldsymbol{L}$ be a lattice. An element $p$ of $\boldsymbol{L}$ is said to be meet-irreducible (MI) in $L$ if, whenever $p=x \wedge y$, then $p=x$ or $p=y$. We say $p$ is meet-prime (MP) in $L$ if, whenever $x \wedge y \leq p$, then $x \leq p$ or $y \leq p$. If $L$ has a top element, T, observe that $p<\mathrm{T}$ in both cases (since $\mathrm{T}=\Lambda \varnothing$ ). When $L$ is distributive, its meet-prime and meet-irreducible elements coincide. The notions of join-irreducible (JI) and join-prime (JP) elements are defined dually. If $\boldsymbol{L}$ is complete, then we obtain the concepts of completely meet-irreducible (CMI) element, completely meet-prime (CMP) element, completely join-irreducible (CJI) element, and completely join-prime (CJP) element by extending the above definitions to arbitrary meets and joins. Note that an element of $L$ is CJP if and only if it is compact and join-prime.

The following well-known abstraction of Birkhoff's subdirect product theorem plays an important role in any discussion concerning algebraic lattices and will be used without explicit reference:

Every element of an algebraic lattice is the meet of a set of CMI elements.

We will use $\operatorname{Com}(L)$ to denote the set of compact elements of an algebraic lattice $L$. It is clear that $L$ is a member of the class $\mathscr{G R N}$ if and only if it is isomorphic to the ideal lattice of some relatively normal lattice. In other words, $\boldsymbol{L}$ is a member of the class $\mathscr{I R N}$ if and only if $\operatorname{Com}(\boldsymbol{L})$ is a distributive lattice and $\operatorname{MP}(\boldsymbol{L})$ is a root-system.

The next result, due essentially to Monteiro $[24,25]$, catalogues several conditions equivalent to relative normality in lower-bounded, distributive lattices.

Theorem 1.1. For a lower-bounded, distributive lattice $\boldsymbol{D}$ the following are equivalent:

(1) D is relatively normal.

(2) Any two incomparable prime ideals in $\boldsymbol{D}$ have disjoint open neighborhoods in $\boldsymbol{\Omega}(\boldsymbol{D})$, the Stone space of $\boldsymbol{D}$.

(3) The join of any pair of incomparable prime filters in $\boldsymbol{D}$ is all of $\boldsymbol{D}$.

(4) For all $a, b \in \boldsymbol{D}$, there exist $p, q \in \boldsymbol{D}$ such that

$$
p \wedge q=\perp \text { and } a \vee q=b \vee p=a \vee b .
$$


The reader is referred to Monteiro [24] or Zaanen [28] for a proof of this result. The element-wise characterization of relative normality given in (4) will play a central role in many of the results to follow.

A lattice is said to be bialgebraic if it is both algebraic and dually algebraic. Snodgrass and Tsinakis have established a characterization of the bialgebraic members of the class $\mathscr{I R N}$ which will play a critical role in the decomposition theorems presented in $\S \S 4$ and 5. Before stating this characterization, we introduce a few preliminary results.

A subset $\boldsymbol{I}$ of a poset $\boldsymbol{P}$ is said to be a lower set of $\boldsymbol{P}$ if, whenever $x \in \boldsymbol{I}$ and $y \leq x$, then $y \in I$. It is clear that, under the operations of set-union and intersection, the set $\mathbf{O}(\boldsymbol{P})$ of all lower sets of $\boldsymbol{P}$ forms a bialgebraic, distributive lattice. The CJP elements of $\mathbf{O}(\boldsymbol{P})$ are the principal lower sets of $\boldsymbol{P}$, with the exception of $\{\perp\}$, if $\perp$ exists. Conversely, if $\boldsymbol{L}$ is a bialgebraic, distributive lattice and $\boldsymbol{P}=\mathbf{C J P}(\boldsymbol{L})$, then $\boldsymbol{L}$ is isomorphic to $\mathbf{O}(\boldsymbol{P})$.

Let $\boldsymbol{L}$ be an algebraic lattice. We say an element $p$ of $\boldsymbol{L}$ is a value of an element $x$ of $\boldsymbol{L}$ provided $p$ is maximal with respect to not exceeding $x$. In other words, $p$ is a value of $x$ provided $x \leq p$ and, if $p<q$, then $x \leq q$. We write $p \in \operatorname{Val}(x)$ in this event. Zorn's Lemma implies that every compact element of $\boldsymbol{L}$ other than $\perp$ has at least one value. It is easy to see that whenever $c, d \in \operatorname{Com}(L)$ and $c<d$, then $\operatorname{Val}(c) \subset \operatorname{Val}(d)$.

It is clear that $p$ is a value of a compact element if and only if $p$ is CMI in $L$. A compact element of $L$ is CJP if and only if it has a unique value; an element of $\boldsymbol{L}$ is CMP if and only if it is the unique value of a compact element. In fact, if $j \in \boldsymbol{L}$ is CJP, then

$$
j_{-}=\bigvee\{x \in L: x \geq j\}
$$

is CMP and is the unique value of $j$. Conversely, if $p \in \boldsymbol{L}$ is CMP, then

$$
p^{+}=\bigwedge\{y \in L: y \not p\}
$$

is CJP; and $p$ is the unique value of $p^{+}$. Furthermore, the mapping $j \mapsto j_{-}$ is an order isomorphism from the poset $\operatorname{CJP}(L)$ to the poset $\operatorname{CMP}(L)$ whose inverse is the mapping $p \mapsto p^{+}$. It follows that if $L$ is a member of the class $\mathscr{G R} \mathcal{N}$, then $\mathbf{C J P}(\boldsymbol{L})$ is a root-system. In fact, the following result is established in Snodgrass and Tsinakis [27].

Lemma 1.2. If $L$ is a member of the class $\mathscr{G R N}$, then $\mathbf{C J P}(L)$ is a root-system whose incomparable elements are orthogonal in $\boldsymbol{L}$.

(Recall that in a lower-bounded lattice, two elements $x$ and $y$ are orthogonal (or disjoint) if $x, y>\perp$ and $x \wedge y=\perp$.)

An algebraic lattice is said to be finite-valued if each of its compact elements has only finitely many values. Snodgrass and Tsinakis have shown that, for members of the class $\mathscr{I R N}$, being finite-valued is equivalent to being bialgebraic. In particular, they have shown the following:

Theorem 1.3. Let $L$ be a member of the class $\mathscr{I R N}$. For a compact element $c$ in $\boldsymbol{L}$, the following are equivalent:

(1) The element $c$ has only finitely many values.

(2) The ideal $\downarrow c$ in $\mathbf{L}$ has finitely many co-atoms.

(3) Every value of $c$ is CMP. 
(4) The element $c$ has a unique representation as the join of a finite set of pairwise orthogonal CJP elements.

The following characterization of bialgebraic lattices in the class $\mathscr{S R N}$ follows easily from Theorem 1.3.

Corollary 1.4. For a lattice $\boldsymbol{L}$, the following are equivalent:

(1) $\boldsymbol{L}$ is bialgebraic and a member of the class $\mathscr{I R N}$.

(2) $\boldsymbol{L}$ is finite-valued and a member of the class $\mathscr{G R N}$.

(3) $\boldsymbol{L}$ is isomorphic to $\mathbf{O}(\boldsymbol{R})$ for some root-system $\boldsymbol{R}$.

\section{ORDINAL EXTENSIONS, ORDINAL ELEMENTS, AND BASES}

In this section, we provide a brief summary of the theory of ordinal extensions developed in Snodgrass and Tsinakis [27]. Unless otherwise noted, all references in this section will be to that paper. While a fragment of the theory presented in this section may be developed in a more general setting, we restrict attention to the class $\mathscr{I R R}$.

We reserve the usual notation $\downarrow x$ to denote the principal lower set $\{y \in$ $\boldsymbol{P}: y \leq x\}$ generated by $x$ in a poset $\boldsymbol{P}$. In addition, we shall use the term node to describe a member of a poset which is comparable to every other member.

Definition 2.1. Let $a, b \in L$ such that $a \leq b$. We say $b$ is an ordinal extension of an element $a \leq b$ provided $\downarrow b \backslash \downarrow a$ is a chain, each element of which exceeds $a$.

We will let $\operatorname{Ord}(a)$ denote the set of all ordinal extensions of $a$. We say $b$ is a proper ordinal extension of $a$ provided $b \in \operatorname{Ord}(a)$ and $a<b$. If $b$ is a proper ordinal extension of some element $a$, we say $b$, is an ordinal element. We say $b$ is a maximal ordinal extension of $a$ provided $b$ is a proper ordinal extension of $a$, and $b$ admits no proper ordinal extension. If $b$ is a maximal ordinal extension of some element $a$, then we say $b$ is a maximal ordinal element.

The relation $\rho$ on $L$ defined by $(a, b) \in \rho \Leftrightarrow b \in \operatorname{Ord}(a)$ defines a partial ordering on $\boldsymbol{L}$.

Lemma 2.2. If $a \leq b$ in an algebraic, distributive lattice $\boldsymbol{L}$, then the following are equivalent:

(1) $b$ is an ordinal extension of $a$.

(2) Every compact member of $\downarrow b \backslash \downarrow a$ is CJP in $L$.

In addition, statements (1) and (2) imply that a is a meet-prime node of $\downarrow b$. If $\boldsymbol{L}$ is a member of the class $\mathscr{Y R \mathscr { N }}$, the converse is also true.

Lemma 2.2 can be used to establish the following critical fact about ordinal elements:

Lemma 2.3. If $\boldsymbol{L}$ is a member of the class $\mathscr{Y R N}$, then two ordinal elements of $\boldsymbol{L}$ are either comparable or orthogonal.

Lemma 2.3 implies, in particular, that the ordinal elements of a member of the class $\mathscr{I R N}$ form a root-system. There are a number of connections between the set of ordinal elements and the Boolean skeleton of members of 
the class $\mathscr{G R} \mathcal{N}$. The following connection will be of considerable use in this paper.

Lemma 2.4. If $\boldsymbol{L}$ is a member of the class $\mathscr{Y R N}$ and $a \in \boldsymbol{L}$ admits a proper ordinal extension $b$, then $b^{* *}$ is a maximal ordinal extension of $a$. Moreover, if $\perp<a$, then the ordinal extensions of a form a chain with largest element $a^{* *}$.

Let $\mathbf{M}(\boldsymbol{L})$ denote the subposet of maximal ordinal elements of a lattice $\boldsymbol{L}$. If $\boldsymbol{L}$ is a member of the class $\mathscr{I R N}$, then two elements of $\mathbf{M}(L)$ are either comparable or orthogonal in $L$; in particular, $\mathbf{M}(\boldsymbol{L})$ is a root-system.

Every CJP element is a proper ordinal extension of the unique element it covers. Consequently, the following description of $\mathbf{M}(\boldsymbol{L})$ is an immediate consequence of Lemmas 2.2 and 2.4.

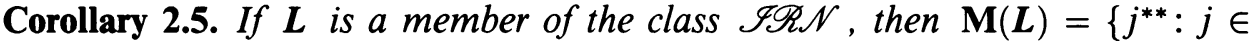
CJP $(L)\}$.

An element $b$ of a lattice $L$ is said to be linear if $\downarrow b$ is a chain. Note that if $\boldsymbol{L}$ is lower-bounded, then $b$ is a linear element if and only if $b \in \operatorname{Ord}(L L)$. A linear element $b$ in a lower-bounded lattice $L$ is proper if $\perp<b$. A linear element $b \in \boldsymbol{L}$ is maximal if no linear element properly exceeds $b$. Note that every proper linear element is an ordinal element; in particular, every proper maximal linear element is a maximal ordinal element. We have the following characterization of linear elements within members of the class $\mathscr{G R N}$.

Lemma 2.6. The following are equivalent for a member $\boldsymbol{L}$ of the class $\mathscr{I R N}$ :

(1) $b$ is a proper linear element of $\boldsymbol{L}$.

(2) $b^{* *}$ is a maximal linear element.

(3) $b^{* *}$ is an atom of the Boolean skeleton of $\boldsymbol{L}$.

(4) $b^{*}$ is meet-prime in $\boldsymbol{L}$.

Let $\boldsymbol{L}$ be a lower-bounded lattice. An orthogonal subset of $\boldsymbol{L}$ is a subset of $\boldsymbol{L} \backslash\{\perp\}$ whose elements are pairwise orthogonal. An easy application of Zorn's Lemma shows that every orthogonal subset of $\boldsymbol{L}$ is contained in a maximal orthogonal subset of $\boldsymbol{L}$. An orthogonal subset $\boldsymbol{S}$ of $\boldsymbol{L}$ is maximal if $x \in \boldsymbol{L} \backslash \boldsymbol{S}$ implies $x \wedge s>\perp$ for some $s \in \boldsymbol{S}$. A subset $\boldsymbol{B}$ of $\boldsymbol{L}$ is a basis for $\boldsymbol{L}$ if $\boldsymbol{B}$ is a maximal orthogonal set consisting of proper linear elements. The following result is an immediate corollary of Lemma 2.6.

Corollary 2.7. The following are equivalent for a member $\boldsymbol{L}$ of the class $\mathscr{I R \mathscr { N }}$ :

(1) L has a basis.

(2) Every element of $\boldsymbol{L}$ exceeds a proper linear element.

(3) The Boolean skeleton of $\boldsymbol{L}$ is atomic.

Observe that if $\boldsymbol{L}$ is a member of the class $\mathscr{Y R N}$ and has a basis, then the atoms of the Boolean skeleton form a basis consisting of maximal linear elements. It is also clear that $\boldsymbol{L}$ has a basis consisting of compact (indeed, CJP) elements and that any two bases have the same cardinality.

\section{Filet CONFigurations}

In this section, we prove that if $D$ is a relatively normal lattice such that the quotient lattice $D / \boldsymbol{\theta}$, relative to the Glivenko congruence $\boldsymbol{\theta}$, satisfies the 
descending chain condition DCC, then the ideal lattice $\mathbf{I}(\boldsymbol{D})$ is bialgebraic. We further characterize the poset of CJP elements of $\mathbf{I}(\boldsymbol{D})$ in terms of forbidden substructures. We commence by introducing some additional notation and terminology.

Let $\boldsymbol{D}$ be a lower-bounded, distributive lattice and let $\boldsymbol{L}=\mathbf{I}(\boldsymbol{D})$. Given $a \in \boldsymbol{D}$, the pseudocomplement of $\downarrow a$ in $\boldsymbol{L}$ will be denoted by $a^{*}$. Note that $a^{*}=\{x \in \boldsymbol{D}: a \wedge x=\perp\}$. We denote by $\boldsymbol{\Theta}$ the Glivenko congruence of $\boldsymbol{D}$ :

$$
\boldsymbol{\Theta}=\left\{(a, b) \in \boldsymbol{D} \times \boldsymbol{D}: a^{* *}=b^{* *}\right\} .
$$

Finally, let $\mathbf{F}(\boldsymbol{L})=\left\{c^{* *}: c \in \operatorname{Com}(\boldsymbol{L})\right\}$. It is immediate that $\mathbf{F}(\boldsymbol{L})$ is a sublattice of $\mathbf{B}(\boldsymbol{L})$. Further, the mapping $a \mapsto a^{* *}$ is a lattice homomorphism from $\boldsymbol{D}$ to $B(L)$ with image $\mathbf{F}(\boldsymbol{L})$ and kernel $\Theta$. Thus, $D / \Theta$ is isomorphic to $\mathbf{F}(\boldsymbol{L})$. We will call $\boldsymbol{D} / \boldsymbol{\Theta}$ the Glivenko quotient of $\boldsymbol{D}$.

Lemma 3.1. Let $\boldsymbol{D}$ be a lower-bounded, distributive lattice. For elements $a<b$ in $\boldsymbol{D}$, the following are equivalent:

(1) $a^{* *} \subset b^{* *}$.

(2) $[a]_{\Theta}<[b]_{\Theta}$.

(3) There exist $\perp<c \in D$ such that $c<b$ and $a \wedge c=\perp$.

Proof. We establish the equivalence of (1) and (3). Suppose that $a^{* *} \subset b^{* *}$. Then $I=\downarrow b \cap a^{*} \neq\{\perp\}$. Any $\perp<c \in I$ satisfies (3). Conversely, if $c$ satisfies (3), then $c \in b^{* *} \backslash a^{* *}$.

A filet configuration (see Figure 1) is a poset $\boldsymbol{F}$ with the following properties:

(1) $\boldsymbol{F}=\left\{a_{n}: n<N\right\} \cup\left\{b_{n}: 1 \leq n<N\right\}$ for some ordinal $0<N \leq \omega$.

(2) For all $1 \leq n<N, a_{n-1}$ is an upper bound for $a_{n}$ and $b_{n}$.

(3) For all $1 \leq n<N, a_{n} \| b_{n}$; that is, $a_{n}$ is incomparable to $b_{n}$.

A subposet $\boldsymbol{F}$ of a poset $\boldsymbol{P}$ is a strong filet configuration provided $\boldsymbol{F}$ is a filet configuration and $a_{n}, b_{n}$ have no common lower bounds in $\boldsymbol{P}$ (with the exception of the least element of $\boldsymbol{P}$, if such exists). In a strong filet configuration, the elements $b_{n}$ are pairwise incomparable. If $\boldsymbol{P}$ is a root-system, then every filet configuration in $\boldsymbol{P}$ is strong. The following is an easy consequence of Lemma 3.1.

Corollary 3.2. If $\boldsymbol{D}$ is a lower-bounded, distributive lattice, then $\boldsymbol{D} / \boldsymbol{\Theta}$ satisfies $D C C$ if and only if every strong filet configuration in $\boldsymbol{D}$ is finite.

We now state and prove the main result of this section.

Theorem 3.3. For a member $\boldsymbol{L}$ of the class $\mathscr{G R N}$, the following are equivalent:

(1) $\operatorname{Com}(L) / \Theta$ satisfies $D C C$.

(2) L is finite-valued (bialgebraic) and $\mathbf{M}(\boldsymbol{L})$ satisfies $D C C$.

(3) $L$ is finite-valued and every filet configuration in $\operatorname{CJP}(\boldsymbol{L})$ is finite.

Proof. The equivalence of (2) and (3) is an immediate consequence of Lemma 3.1 and Corollary 3.2. We prove that (1) implies (2) and (3) implies (1).

(1) $\Rightarrow(2)$ Since $\mathbf{M}(\boldsymbol{L})=\left\{j^{* *}: j \in \mathbf{C J P}(\boldsymbol{L})\right\}$, Lemma 3.1 implies $\mathbf{M}(\boldsymbol{L})$ satisfies DCC. Suppose, by way of contradiction, that there exist $\perp<c \in \operatorname{Com}(\boldsymbol{L})$ such that $\operatorname{Val}(c)$ is infinite. By Theorem 1.3, there exist $p \in \operatorname{Val}(c)$ which is not CMP. 
Let $r \in \operatorname{Val}(c) \backslash\{p\}$. Since $\operatorname{Com}(L)$ is a relatively normal lattice and $r \| p$, we know by Theorem 1.1(4) that there exist $\perp<b, d \in \operatorname{Com}(L)$ such that $b \wedge d=\perp$ and $p \vee d=r \vee b=p \vee r$. Let $a_{0}=c, b_{1}=c \wedge d$, and $a_{1}=c \wedge b$. Then $\perp<a_{1}, b_{1} \in \operatorname{Com}(L)$, and $a_{1} \wedge b_{1}=\perp$. Since $a_{1} \vee b_{1} \leq a_{0}$, it follows that $\boldsymbol{F}=\left\{a_{0}, a_{1}\right\} \cup\left\{b_{1}\right\}$ is a strong filet configuration in $\operatorname{Com}(\boldsymbol{L})$.

Since $p \in \operatorname{Val}(c), a_{1} \leq c$, and $a_{1} \leq \leq p$, it follows that $p \in \operatorname{Val}\left(a_{1}\right)$. Hence, $\operatorname{Val}\left(a_{1}\right)$ is infinite by Theorem 1.3. We may therefore repeat this process indefinitely to create an infinite strong filet configuration $\boldsymbol{F}=\left\{a_{n}: n<\omega\right\} \cup\left\{b_{n}: 1 \leq\right.$ $n<\omega\}$ in $\operatorname{Com}(\boldsymbol{L})$, contradicting Corollary 3.2.

(3) $\Rightarrow(1)$ For a proof by contradiction, suppose $\operatorname{Com}(L) / \Theta$ fails to satisfy DCC. By Corollary 3.2, $\operatorname{Com}(L)$ contains an infinite strong filet configuration $\boldsymbol{F}=\left\{a_{n}: n<\omega\right\} \cup\left\{b_{n}: 1 \leq n<\omega\right\}$. We will use this fact to contradict the assumption that every filet configuration in $\operatorname{CJP}(\boldsymbol{L})$ is finite.

Observe that $\boldsymbol{L}$ is bialgebraic by Corollary 1.4. We may therefore assume that each $b_{n}$ is CJP and that $a_{0}$ has a representation as a finite join of pairwise orthogonal CJP elements. It follows that there exists a CJP element $j_{1} \leq a_{0}$ which exceeds infinitely many of the elements $b_{n}$. If $\left\{b_{k_{n}}: 1 \leq n<\omega\right\}$ denotes the set of elements $b_{n}$ exceeded by $j_{1}$, then it is easily seen that

$$
\boldsymbol{F}_{1}=\left\{j_{1}, j_{1} \wedge a_{k_{1}}, j_{1} \wedge a_{k_{2}}, \ldots\right\} \cup\left\{b_{k_{n}}: 1 \leq n<\omega\right\}
$$

is an infinite strong filet configuration in $\operatorname{Com}(L)$. This process may be repeated with $j_{1} \wedge a_{k_{1}}$ in place of $a_{0}$. Thus, an inductive argument establishes the existence of an infinite filet configuration in $\operatorname{CJP}(\boldsymbol{L})$, contradicting condition (3).

The following result establishes the equivalence of statements (1) and (2) in Theorem B and is a consequence of Corollary 1.4 and Theorem 3.3.

Corollary 3.4. The following conditions are equivalent in an algebraic, distributive lattice $\boldsymbol{L}$ :

(1) $L$ is a member of the class $\mathscr{Y R N}$ and $\operatorname{Com}(\boldsymbol{L}) / \boldsymbol{\Theta}$ satisfies DCC.

(2) $\boldsymbol{L}$ is isomorphic to $\mathbf{O}(\boldsymbol{R})$ for some root-system $\boldsymbol{R}$ in which every filet configuration is finite.

Corollary 3.5. If $\boldsymbol{L}$ is a member of the class $\mathscr{Y R N}$ and satisfies the conditions of Theorem 3.3, then $\mathbf{L}$ has a basis.

Proof. By Corollary 2.7, it suffices to show the Boolean skeleton $\mathbf{B}(\boldsymbol{L})$ is atomic. If $a \in \mathbf{B}(\boldsymbol{L})$, then $a=x^{* *}$ for some $x \in \boldsymbol{L}$. Since $\boldsymbol{L}$ is bialgebraic, there exist $j \in \mathbf{C J P}(L)$ such that $j^{* *} \leq x^{* *}$. It is easy to see that the least element of any descending chain in $\mathbf{M}(\boldsymbol{L})$ with top element $j^{* *}$ is an atom of $\mathbf{B}(\boldsymbol{L})$.

We conclude this section by presenting some additional results concerning the structure of the Glivenko quotient of a lower-bounded, distributive lattice. First, we recall a few basic facts and definitions.

A generalized Boolean lattice is a relatively complemented, distributive lattice with least element. A poset $\boldsymbol{P}$ satisfies the weak ascending chain condition WACC if every upper-bounded strictly ascending chain in $\boldsymbol{P}$ is finite. A lowerbounded, distributive lattice $\boldsymbol{D}$ is called a disjunctive lattice if, whenever $a, b \in$ $D$ and $a<b$, there exist $\perp<c \in \boldsymbol{D}$ such that $c<b$ and $a \wedge c=\perp$. It is clear in light of Lemma 3.1 that the Glivenko quotient of every lower-bounded, distributive lattice is a disjunctive lattice. 
Lemma 3.6. For a disjunctive lattice $\boldsymbol{D}$, the following are equivalent:

(1) D satisfies WACC.

(2) D is a generalized Boolean lattice satisfying DCC.

(3) D is isomorphic to the generalized Boolean lattice of all finite subsets of some set.

Proof. (1) $\Rightarrow(2)$ Suppose condition (1) is satisfied. We first show that $\boldsymbol{D}$ is a generalized Boolean lattice. Suppose, by way of contradiction, that there exists a $b \in \boldsymbol{D}$ such that $\downarrow b$ is not a Boolean lattice.

There exists $\perp<a<b$ in $\boldsymbol{D}$ such that $a$ has no complement in $\downarrow b$. Since $\boldsymbol{D}$ is disjunctive, there exists $\perp<b_{1}<b$ in $\boldsymbol{D}$ such that $a \wedge b_{1}=\perp$. Since $a$ has no complement in $\downarrow b$, it follows that $a_{1}=a \vee b_{1}<b$.

Note that $a<a_{1}<b$. There exist $\perp<b_{2}<b$ in $\boldsymbol{D}$ such that $a_{1} \wedge b_{2}=\perp$. If $a_{1}$ has a complement, $d$, in $\downarrow b$, then $b_{1} \vee d$ is a complement for $a$ in $\downarrow b$, contrary to assumption. Consequently, $a_{2}=a_{1} \vee b_{2}<b$. An inductive argument now establishes the existence of an infinite strictly ascending chain $a<a_{1}<a_{2} \cdots$ bounded above by $b$, contradicting condition (1).

We have shown that $\boldsymbol{D}$ is a generalized Boolean lattice. To prove that $\boldsymbol{D}$ satisfies DCC, it suffices to show that, for all $b \in \boldsymbol{D}, \downarrow b$ satisfies DCC. This, however, is immediate since $\downarrow b$ is a Boolean lattice and satisfies ACC by condition (1).

$(2) \Rightarrow(3)$ Suppose that condition (2) is satisfied. It is clear that $\boldsymbol{D}$ is atomic. Let $\perp<b$ be an element of $\boldsymbol{D}$ and let $\operatorname{At}(b)$ denote the set of atoms below $b$. We first prove that $\operatorname{At}(b)$ is finite.

Suppose by way of contradiction that $\mathbf{A t}(b)$ contains a countably infinite subset $\left\{x_{n}: n<\omega\right\}$. This set yields an infinite strictly ascending chain $x_{0}<$ $\left(x_{0} \vee x_{1}\right)<\cdots<\left(x_{0} \vee x_{1} \vee \cdots \vee x_{n}\right)<\cdots$ in $\downarrow b$, contradicting condition (3). Thus, $\operatorname{At}(b)$ is finite.

Let $\boldsymbol{X}$ denote the set of atoms of $\boldsymbol{D}$. The map $b \mapsto \boldsymbol{A t}(b)$ provides the desired isomorphism of $\boldsymbol{D}$ onto the generalized Boolean algebra of all finite subsets of $\boldsymbol{X}$.

(3) $\Rightarrow$ (1) Immediate.

A lower-bounded, distributive lattice $\boldsymbol{D}$ is orthofinite provided every orthogonal, upper-bounded subset of $\boldsymbol{D}$ is finite.

Lemma 3.7. The Glivenko quotient of lower-bounded, distributive lattice $\boldsymbol{D}$ satisfies the conditions of Lemma 3.6 if and only if $\boldsymbol{D}$ is orthofinite.

Proof. Suppose $\boldsymbol{D}$ is orthofinite. Let $\boldsymbol{L}$ denote the ideal lattice of $\boldsymbol{D}$ and recall that $\boldsymbol{D} / \boldsymbol{\Theta}$ is isomorphic to $\mathbf{F}(\boldsymbol{L})=\left\{a^{* *}: a \in \boldsymbol{D}\right\}$. Assume, by way of contradiction, that $\mathbf{F}(\boldsymbol{L})$ fails to satisfy WACC. Then there exists an upper bound $b^{* *} \in \mathbf{F}(\boldsymbol{L})$ for an infinite ascending chain $a_{0}^{* *}<a_{1}^{* *}<\cdots$ in $\mathbf{F}(\boldsymbol{L})$. Clearly, we may assume $a_{n}<a_{n+1}<b$ in $\boldsymbol{D}$ for all $n$; hence, by Lemma 3.1, there exist $\perp<b_{n}<a_{n+1}$ such that $a_{n} \wedge b_{n}=\perp$. It follows that $b$ is an upper bound for the infinite orthogonal set $\left\{b_{n}: n<\omega\right\}$, a contradiction.

Conversely, suppose $\mathbf{F}(\boldsymbol{L})$ satisfies the conditions of Lemma 3.6. Then $\mathbf{F}(\boldsymbol{L})$ is a generalized Boolean lattice and satisfies WACC. Suppose, by way of contradiction, that there exist $b \in \boldsymbol{D}$ which exceeds a countably infinite orthogonal set $\left\{x_{0}, x_{1}, \ldots\right\}$. It is clear that $x_{n}^{* *} \wedge x_{m}^{* *}=\perp$ if $n \neq m$. Consequently, $x_{0}^{* *}<\left(x_{0} \vee x_{1}\right)^{* *}<\cdots<\left(x_{0} \vee \cdots \vee x_{n}\right)^{* *}<\cdots$ forms an infinite ascending 
chain in $\downarrow b^{* *} \cap \mathbf{F}(\boldsymbol{L})$-an impossibility since $\downarrow b^{* *} \cap \mathbf{F}(\boldsymbol{L})$ is a Boolean lattice which satisfies ACC.

Lemma 3.7, while not necessary for the development of the decomposition theory to follow, provides additional insight into the structure of some of the lattices considered in $\S 5$. (See, in particular, Theorems 5.8, 5.9, and 5.10.)

\section{ORDINAL DECOMPOSITIONS}

The goal of this section will be to complete the proof of Theorem B. We begin by introducing the notion of an ordinal decomposition.

Definition 4.1. Let $\boldsymbol{L}$ be a complete lattice and let $g \in \boldsymbol{L}$. We say $g$ admits an ordinal decomposition provided there exists an ordinal $0<\delta$ and an ascending chain $\left\{z^{\beta}: \beta<\delta\right\}$ of elements of $\boldsymbol{L}$ such that

(1) $g=\bigvee\left\{z^{\beta}: \beta<\delta\right\}$,

(2) $z^{\beta}=\bigvee \mathbf{B}(\beta)$, where $\mathbf{B}(\beta)=\left\{b_{j}^{\beta}: j \in J_{\beta}\right\}$ is an orthogonal set, and

(3) if $\beta$ is a successor ordinal, then each $b_{j}^{\beta}$ is either a joinand of $z^{\beta-1}$ or else a proper ordinal extension of the join of two or more joinands of $z^{\beta-1}$;

(4) if $0<\beta$ is a limit ordinal, then $b_{j}^{\beta}$ is a (possibly trivial) ordinal extension of the join of a transfinite sequence $\boldsymbol{W}_{j}^{\beta}$ of elements $w_{j}^{0} \leq$ $w_{j}^{1} \leq \cdots \leq w_{j}^{\xi} \leq \cdots$, one chosen from each $\mathbf{B}(\xi), 0 \leq \xi<\beta$.

We shall refer to the sets $\mathbf{B}(\beta)=\left\{b_{j}^{\beta}: j \in J_{\beta}\right\}$ as $\beta$-levels. If we wish to emphasize the initial level $\mathbf{B}(0)$, then we will refer to the ordinal decomposition over the set $\mathbf{B}(0)$.

The first goal of this section will be to devise a method by which ordinal decompositions may be constructed within members of the class $\mathscr{S R}$. Let $\boldsymbol{L}$ be a member of the class $\mathscr{I R N}$ and set

$$
\mathbf{C M}(\boldsymbol{L})=\{x \in L: x=\bigvee \boldsymbol{C} \text { for some chain } \boldsymbol{C} \text { in } \mathbf{M}(\boldsymbol{L})\} .
$$

The following results concerning the set $\mathbf{C M}(L)$ tacitly assume that $L$ is a member of the class $\mathscr{I R N}$.

Lemma 4.2. $\mathbf{C M}(L)$ is a root-system in which incomparable elements are orthogonal in $\mathbf{L}$.

Proof. Let $x, y \in \mathbf{C M}(\boldsymbol{L})$. There exist chains $C_{1}, C_{2}$ such that $x=\bigvee C_{1}$ and $y=\bigvee C_{2}$. Suppose, by way of contradiction, that $x \| y$ but $\perp<x \wedge y$. Since $L$ satisfies JID, there exist elements $c_{1} \in C_{1}$ and $c_{2} \in C_{2}$ such that $\perp<c_{1} \wedge c_{2}$. It follows that $c_{1}$ and $c_{2}$ are comparable; say, $c_{1} \leq c_{2}$. Since $x \| y$, there exist incomparable elements $c_{1}^{\prime} \in C_{1}$ and $c_{2}^{\prime} \in C_{2}$ exceeding $c_{1}$ and $c_{2}$, respectively. Thus, $c_{1}$ is a lower bound for $c_{1}^{\prime}$ and $c_{2}^{\prime}$-an impossibility since $\mathbf{M}(\boldsymbol{L})$ is a root-system.

Lemma 4.3. If $x<y$ in $\mathbf{C M}(L)$, then there exist $z \in \mathbf{M}(L)$ such that $x<z \leq$ $y$.

Proof. Let $x=\bigvee C_{1}$ and $y=\bigvee C_{2}$, where $C_{1}$ and $C_{2}$ are chains in $\mathbf{M}(\boldsymbol{L})$. Since $x<y$, there exists by JID a $z \in C_{2}$ such that $z \not x$ and $\perp<x \wedge z$. It follows that $c<z$ for each $c \in C_{1}$; consequently, $x<z$. 
Clearly, $\mathbf{M}(\boldsymbol{L}) \subseteq \mathbf{C M}(\boldsymbol{L})$. An interesting consequence of Lemma 4.3 is that $\mathbf{C M}(L)$ is chain-complete; that is, every chain in $\mathbf{C M}(\boldsymbol{L})$ has a least upper bound in $\mathbf{C M}(\boldsymbol{L})$, namely the join of the chain (in $L$ ). In fact, using the notion of the chain-completion of a poset as defined in Markowsky and Rosen [21], we may say even more about $\mathbf{C M}(\boldsymbol{L})$.

Corollary 4.4. $\mathrm{CM}(\boldsymbol{L})$ is the chain-completion of the root-system $\mathbf{M}(\boldsymbol{L})$.

We will prove that $\mathbf{C M}(\boldsymbol{L})$ is a source of ordinal decompositions within members $\boldsymbol{L}$ of the class $\mathscr{G R} \mathscr{N}$. The following result will be crucial to this endeavor. It shows, in particular, that if $b$ is an element of a member $\boldsymbol{L}$ of the class $\mathscr{I R \mathscr { N }}$, then there is at most one orthogonal subset $\boldsymbol{B}$ in $\mathbf{C M}(\boldsymbol{L})$ such that $b$ is a proper ordinal extension of $\bigvee \boldsymbol{B}$.

Lemma 4.5. Let $\mathrm{B} \subseteq \mathrm{CM}(\boldsymbol{L})$ be a nonempty orthogonal set. Let $\boldsymbol{B}_{1}$ and $\boldsymbol{B}_{2}$ be nonempty subsets of $\boldsymbol{B}$ and let $b_{1}$ and $b_{2}$ be proper ordinal extensions of $\bigvee \boldsymbol{B}_{1}$ and $\bigvee B_{2}$, respectively.

(1) $b_{1} \wedge b=\perp$ for all $b \in \boldsymbol{B} \backslash \boldsymbol{B}_{1}$.

(2) If $\boldsymbol{B}_{1} \nsubseteq \boldsymbol{B}_{2}$ and $\boldsymbol{B}_{2} \nsubseteq \boldsymbol{B}_{1}$, then $b_{1} \wedge b_{2}=\perp$ and $\boldsymbol{B}_{1} \cap \boldsymbol{B}_{2}=\varnothing$.

(3) If $\boldsymbol{B}_{1} \subseteq \boldsymbol{B}_{2}$, then $b_{1}$ is comparable to $b_{2}$ and $\boldsymbol{B}_{1}=\boldsymbol{B}_{2}$. Furthermore, if $b_{1}, b_{2} \in \mathbf{M}(\boldsymbol{L})$, then $b_{1}=b_{2}$.

Proof. (1) Let $b \in \boldsymbol{B} \backslash \boldsymbol{B}_{1}$. In light of Lemmas 2.4 and 4.2 , it suffices to prove that $b_{1}^{* *} \| b$. It is clear that $b_{1}^{* *} \not \leq b$. Since $\boldsymbol{B}$ is an orthogonal set and $\boldsymbol{L}$ satisfies JID, $\bigvee \boldsymbol{B}_{1} \wedge b=\perp$. It thus follows from Lemma 2.2 that $b \not b_{1}^{* *}$.

(2) Observe JID implies that, whenever $\boldsymbol{C} \subset \boldsymbol{D} \subseteq \boldsymbol{B}$, then $\bigvee \boldsymbol{C}<\bigvee \boldsymbol{D}$. Suppose now that the assumption of statement (2) is satisfied, but $\perp<b_{1} \wedge b_{2}$. By Lemma 2.3, we may assume $b_{1} \leq b_{2}$; hence, statement (1) and JID yield

$$
\bigvee \boldsymbol{B}_{1}=\bigvee \boldsymbol{B}_{1} \wedge b_{2}=\bigvee\left\{b \wedge b_{2}: b \in \boldsymbol{B}_{1} \cap \boldsymbol{B}_{2}\right\}=\bigvee\left(\boldsymbol{B}_{1} \cap \boldsymbol{B}_{2}\right)<\bigvee \boldsymbol{B}_{1}
$$

This is an obvious contradiction; hence, we have shown $b_{1} \wedge b_{2}=\perp$. It is now clear that $\boldsymbol{B}_{1} \cap \boldsymbol{B}_{2}=\varnothing$.

(3) Suppose $\boldsymbol{B}_{1} \subseteq \boldsymbol{B}_{2}$; this implies $\bigvee \boldsymbol{B}_{1} \leq \bigvee \boldsymbol{B}_{2}$. Consequently, $\perp<b_{1} \wedge b_{2}$; and $b_{1}$ is therefore comparable to $b_{2}$ by Lemma 2.3. Suppose, by way of contradiction, that there exists a $b^{\prime} \in \boldsymbol{B}_{2} \backslash \boldsymbol{B}_{1}$. Since $\boldsymbol{B}$ is an orthogonal set, we have $b^{\prime} \wedge \bigvee \boldsymbol{B}_{1}=\perp$ by JID; it follows that $b_{1}<\bigvee \boldsymbol{B}_{2}$. By Lemma 2.2 , there exists a CJP element $j$ such that $\bigvee \boldsymbol{B}_{1}<j \leq b_{1}<\bigvee \boldsymbol{B}_{2}$. Hence, there exists $b \in \boldsymbol{B}_{2} \backslash \boldsymbol{B}_{1}$ such that $j \leq b$. This, however, implies $\perp<j \leq b_{1} \wedge b$, which contradicts statement (1). Hence, $\boldsymbol{B}_{1}=\boldsymbol{B}_{2}$.

To complete the proof, note that $\boldsymbol{B}_{1}=\boldsymbol{B}_{2}$ implies $b_{1} \in \operatorname{Ord}\left(b_{2}\right)$ or $b_{2} \in$ $\operatorname{Ord}\left(b_{1}\right)$. Thus, if $b_{1}, b_{2} \in \mathbf{M}(\boldsymbol{L})$, we must have $b_{1}=b_{2}$.

Let $\boldsymbol{L}$ be a member of the class $\mathscr{I R \mathscr { N }}$ and let $\boldsymbol{B}$ be a nonempty orthogonal subset of $\mathbf{C M}(\boldsymbol{L})$. Define a binary relation $\sim_{B}$ on $\boldsymbol{B}$ as follows: $x \sim_{B} y$ if and only if

(1) $x=y$, or

(2) There exists a subset $\boldsymbol{X}$ of $\boldsymbol{B}$ containing $x$ and $y$ such that $\bigvee \boldsymbol{X}$ admits a proper ordinal extension.

Statements (2) and (3) of Lemma 4.5 ensure that $\sim_{B}$ is an equivalence relation. For any $b \in \boldsymbol{B}$, let $[b]_{\sim_{B}}$ denote the equivalence class of $b$ with respect to $\sim_{B}$. 
Now, let $\boldsymbol{L}$ be a member of the class $\mathscr{I R N}$, suppose that $0<\beta$ is a limit ordinal, and suppose that, for each $0 \leq \xi<\beta, \mathbf{B}(\xi) \subseteq \mathbf{C M}(L)$ is the $\xi$-level of an ordinal decomposition in $L$. Let $\mathscr{W}$ denote the set of all chains $W^{\beta} \subseteq \bigcup\{\mathbf{B}(\xi): 0 \leq \xi<\beta\}$ such that $W^{\beta} \cap \mathbf{B}(\xi) \neq \varnothing$ for all $0 \leq \xi<\beta$ and let $\mathscr{W}^{\prime}=\left\{w \in \boldsymbol{L}: w=\bigvee \boldsymbol{W}^{\beta}\right.$ for some $\left.\boldsymbol{W}^{\beta} \in \mathscr{W}\right\}$. For each $w \in \mathscr{W}^{\prime}$, let $b^{\beta}=w$ if $w$ admits no proper ordinal extension; else, let $b^{\beta}=w^{* *}$. Let $\mathbf{B}(\beta)$ denote the set of all such elements $b^{\beta}$.

Lemma 4.6. If $\beta$ is a limit ordinal and $\mathbf{B}(\beta)$ is constructed as above, then $\mathbf{B}(\beta)$ is an orthogonal subset of $\mathbf{C M}(\boldsymbol{L})$.

Proof. Let $b_{1}, b_{2}$ be distinct members of $\mathbf{B}(\beta)$. By construction, $\mathbf{B}(\beta) \subseteq$ $\operatorname{CM}(\boldsymbol{L})$; hence, by Lemma 4.2, it will suffice to show $b_{1} \| b_{2}$. For $j \in\{1,2\}$, there exists a chain $\boldsymbol{W}_{j} \in \mathscr{W}$ such that $b_{j}=\bigvee \boldsymbol{W}_{j}$ or $b_{j}=\left(\bigvee \boldsymbol{W}_{j}\right)^{* *}$. It will therefore suffice to show that $\bigvee W_{1} \wedge \bigvee W_{2}=\perp$ as this implies $\perp=\left(\bigvee W_{1}\right)^{* *} \wedge$ $\left(\bigvee \boldsymbol{W}_{2}\right)^{* *} \geq b_{1} \wedge b_{2}$.

Let $\boldsymbol{W}_{j}=\left\{b_{j}^{\xi}: 0 \leq \xi<\beta\right\}$. By JID, we have

$$
\bigvee \boldsymbol{W}_{1} \wedge \bigvee \boldsymbol{W}_{2}=\bigvee\left\{b_{1}^{\xi} \wedge b_{2}^{\xi}: 0 \leq \xi<\beta\right\}
$$

Since $\mathbf{B}(\xi)$ is an orthogonal set by assumption, either $b_{1}^{\xi}=b_{2}^{\xi}$ or $b_{1}^{\xi} \wedge b_{2}^{\xi}=\perp$ for each $0 \leq \xi<\beta$. If there exists an $\eta<\beta$ such that $b_{1}^{\eta}=b_{2}^{\eta}$, then $b_{1}^{\xi}=b_{2}^{\xi}$ for all $\eta \leq \xi<\beta$. This implies $\bigvee \boldsymbol{W}_{1}=\bigvee \boldsymbol{W}_{2}$ which implies $b_{1}=b_{2}$, contrary to assumption. Thus, $b_{1}^{\xi} \wedge b_{2}^{\xi}=\perp$ for all $0 \leq \xi<\beta$; it is now clear that $\bigvee \boldsymbol{W}_{1} \wedge \bigvee \boldsymbol{W}_{2}=\perp$.

We now detail a method by which $\beta$-levels of an ordinal decomposition may be constructed within a member of the class $\mathscr{I R N}$.

Construction 4.7. Let $\boldsymbol{L}$ be a member of the class $\mathscr{Y R N}$ and let $B$ be a nonempty, maximal orthogonal subset of $\mathbf{M}(\boldsymbol{L})$. For an ordinal $0<\delta$, construct $\beta$-levels $\mathbf{B}(\beta), \beta<\delta$, as follows:

(1) Let $\mathbf{B}(0)=\boldsymbol{B}$.

(2) If $\beta$ is a successor ordinal, let $b^{\beta} \in \mathbf{B}(\beta)$ if and only if $b^{\beta} \in \mathbf{B}(\beta-1)$ and $\left[b^{\beta}\right]_{\sim_{B(\beta-1)}}$ is a singleton, or else $b^{\beta}=\left(\bigvee[b]_{\sim_{B(\beta-1)}}\right)^{* *}$ for some $b \in \mathbf{B}(\beta-1)$.

(3) If $0<\beta$ is a limit ordinal, let $b^{\beta} \in \mathbf{B}(\beta)$ if and only if there exists a chain $\boldsymbol{W}^{\beta} \subseteq \bigcup\{\mathbf{B}(\xi): 0 \leq \xi<\beta\}$, with $\boldsymbol{W}^{\beta} \cap \mathbf{B}(\xi) \neq \varnothing$ for all $0 \leq \xi<\beta$, such that $b^{\beta}=\bigvee W^{\beta}$ if this join admits no proper ordinal extension and $b^{\beta}=\left(\bigvee W^{\beta}\right)^{* *}$ otherwise.

It is clear in light of Lemmas 2.4 and 4.5 that if $\mathbf{B}(\beta)$ is an orthogonal subset of $\mathbf{C M}(\boldsymbol{L})$, then the set $\mathbf{B}(\beta+1)$ as described in Construction 4.7(2) is also an orthogonal subset of $\mathbf{C M}(\boldsymbol{L})$. Utilizing Lemma 4.6 and transfinite induction, one may therefore verify that the sets $B(\beta)$ described in Construction 4.7 are orthogonal subsets of $\mathbf{C M}(L)$ for all $0 \leq \beta<\delta$. In particular, these sets satisfy the conditions of Definition 4.1 and thus form the $\beta$-levels of an ordinal decomposition in $L$. Furthermore, Lemma 4.5(3) implies the elements of each $\beta$-level are uniquely determined. Moreover, since $\mathbf{B}(0)$ is a maximal orthogonal set, so also is each level $\mathbf{B}(\beta), \beta<\delta$. 
We now proceed to complete the proof of Theorem B. We shall have need of the following lemma which provides additional information about the structure of ordinal decompositions.

Lemma 4.8. Suppose $\boldsymbol{L}$ is a member of the class $\mathscr{I R N}$ and $g \in \boldsymbol{L}$ admits an ordinal decomposition over an orthogonal set of proper linear elements as described in Definition 4.1. If $j$ is CJP in $\boldsymbol{L}$ and such that $j \leq z^{\beta}$ but $j \leq z^{\xi}$ for any $0 \leq \xi<\beta$, then there exists an element $b \in \mathbf{B}(\beta)$ such that $j \leq b \leq j^{* *}$. In particular, if $b$ admits no proper ordinal extension, then $b=j^{* *}$.

Proof. Since $j \in \mathbf{C J P}(\boldsymbol{L})$ and $j \leq z^{\beta}=\bigvee \mathbf{B}(\beta)$, there exist $b \in \mathbf{B}(\beta)$ such that $j \leq b$. Since $j^{* *}$ is the largest ordinal extension of $j$ by Lemma 2.4, the desired result will follow once we have shown $b \in \operatorname{Ord}(j)$.

If $\beta=0$, then $b$ is linear by assumption; hence, $b \in \operatorname{Ord}(j)$. Suppose $0<\beta$. Since $j \not \leq z^{\xi}$ for any $0 \leq \xi<\beta$, it follows that $b \notin \mathbf{B}(\xi)$ for any $0 \leq \xi<\beta$. Hence, $b \in \operatorname{Ord}(a)$, where $a$ is constructed in one of two ways. If $\beta$ is a successor ordinal, then $a=\bigvee D$, where $D$ is a subset of $\mathbf{b}(\beta-1)$ containing at least two elements. If $\beta$ is a limit ordinal, then $a=\bigvee \boldsymbol{W}$, where $\boldsymbol{W} \subseteq \bigcup\{\mathbf{B}(\xi): \xi<\beta\}$ is a chain such that $\boldsymbol{W} \cap \mathbf{B}(\xi) \neq \varnothing$ for all $0 \leq \xi<\beta$. Now, $a$ is a node of $\downarrow b$ by Lemma 2.2; hence, $j$ must be comparable to $a$. If $j \leq a$, then by the construction of $a$, there exist $\xi<\beta$ and $b^{\xi} \leq a$ in $\mathbf{B}(\xi)$ such that $j \leq b^{\xi}$. This implies $j \leq z^{\xi}$, contradicting our choice of $j$. Consequently, $a<j \leq b$; it follows that $b \in \operatorname{Ord}(j)$.

Corollary 4.9. Suppose $\boldsymbol{L}$ is a member of the class $\mathscr{G R N}$ whose top element admits an ordinal decomposition over an orthogonal set of maximal linear elements where each $\beta$-level is as described in Construction 4.7. If $g \in \mathbf{M}(\boldsymbol{L})$, then there exists an ordinal $\beta$ such that $g \in \mathbf{B}(\beta)$.

Proof. Let $g \in \mathbf{M}(\boldsymbol{L})$. By Corollary 2.5, there exists a CJP element $j$ such that $g=j^{* *}$. Since $\mathrm{T}=\bigvee\left\{z^{\beta}: \beta<\delta\right\}$, there exists a least ordinal $\beta<\delta$ such that $j \leq z^{\beta}$. By Lemma 4.8, there exist $b^{\beta} \in \mathbf{B}(\beta)$ such that $j \leq b^{\beta} \leq g$. Since $b^{\beta}$ admits no proper ordinal extension, $b^{\beta}=g$.

Lemma 4.10. If $\boldsymbol{L}$ is an algebraic, distributive lattice whose top element admits an ordinal decomposition over an orthogonal set of proper linear elements, then $\boldsymbol{L}$ is a bialgebraic member of the class $\mathscr{G R N}$. Furthermore, $\mathbf{M}(\boldsymbol{L})$ satisfies DCC.

Proof. Let $\mathrm{T}=\bigvee\left\{z^{\beta}: \beta<\delta\right\}$, where each $z^{\beta}=\bigvee \mathbf{B}(\beta)$ is as in Definition 4.1, and where $\mathbf{B}(0)$ is an orthogonal set of proper linear elements. We prove that $L$ is bialgebraic by showing every $c \in \operatorname{Com}(L)$ is a (finite) join of CJP elements. This is accomplished by transfinite induction.

Let $\perp<c \in \operatorname{Com}(\boldsymbol{L})$. There exists a least ordinal $\beta<\delta$ such that $c \leq z^{\beta}$. Suppose $c \leq z^{0}=\bigvee \mathbf{B}(0)$. By JID we have

$$
c=\bigvee\left\{c \wedge b_{j}^{0}: j \in J_{0}\right\}
$$

Since $b_{j}^{0}$ is a linear element and $c \wedge b_{j}^{0} \leq b_{j}^{0}$ for all $j \in J_{0}$, it follows by Lemma 2.2 that $c \wedge b_{j}^{0}$ is the join of a (possibly empty) set of CJP elements. Thus, in this case, $c$ is a join of CJP elements.

Now suppose $c \leq z^{\beta}=\bigvee \mathbf{B}(\beta)$ for some ordinal $0<\beta<\delta$ and suppose that, for all $0 \leq \xi<\beta$, every compact element below $z^{\xi}$ is a join of CJP 
elements. We assume $\beta$ is minimal; that is, $c \leq z^{\beta}$ and $c\left\lfloor z^{\xi}\right.$ for any $0 \leq \xi<\beta$. By JID we have

$$
c=\bigvee\left\{c \wedge b_{j}^{\beta}: j \in J_{\beta}\right\}
$$

Consequently, it will suffice to show $c \wedge b_{j}^{\beta}$ is a join of CJP elements for each $j \in J_{\beta}$. Let $j \in J_{\beta}$ such that $c \wedge b_{j}^{\beta}>\perp$.

We first consider the case when $\beta$ is a successor ordinal. If $b_{j}^{\beta} \in \mathbf{B}(\xi)$ for some $\xi<\beta$, then $c \wedge b_{j}^{\beta} \leq z^{\xi}$. It follows by the induction hypothesis that every compact element below $c \wedge b_{j}^{\beta}$, and thus $c \wedge b_{j}^{\beta}$, is a join of CJP elements. If $b_{j}^{\beta} \notin \mathbf{B}(\xi)$ for all $0 \leq \xi<\beta$, then there is a set $D \subseteq \mathbf{B}(\beta-1)$ containing at least two elements such that $b_{j}^{\beta}$ is a proper ordinal extension of $\vee D$. We know that $\bigvee D$ is a node of $\downarrow b_{j}^{\beta}$ by Lemma 2.2, and hence must be comparable to $c \wedge b_{j}^{\beta}$. If $\vee D<c \wedge b_{j}^{\beta} \leq b_{j}^{\beta}$, then $c \wedge b_{j}^{\beta}$ is a join of CJP elements by Lemma 2.2. If $c \wedge b_{j}^{\beta} \leq \bigvee \boldsymbol{D} \leq z^{\beta-1}$, then every compact element below $c \wedge b_{j}^{\beta}$ is a join of CJP elements by the induction hypothesis. Therefore, $c \wedge b_{j}^{\beta}$ is a join of CJP elements.

We now consider the case when $\beta$ is a limit ordinal. There is a chain $\boldsymbol{W}_{j}^{\beta}=$ $\left\{w_{j}^{\xi}: 0 \leq \xi<\beta\right\}$ as in Definition 4.1 such that $b_{j}^{\beta} \in \operatorname{Ord}\left(\bigvee \boldsymbol{W}_{j}^{\beta}\right)$. We know that $\vee \boldsymbol{W}_{j}^{\beta}$ is a node of $\downarrow b_{j}^{\beta}$. If $\vee \boldsymbol{W}_{j}^{\beta}<c \wedge b_{j}^{\beta} \leq b_{j}^{\beta}$, then $c \wedge b_{j}^{\beta}$ is a join of CJP elements by Lemma 2.2. On the other hand, if $c \wedge b_{j}^{\beta} \leq \bigvee \boldsymbol{W}_{j}^{\beta}$, then every compact element below $c \wedge b_{j}^{\beta}$ is below some $w_{j}^{\xi} \in \boldsymbol{W}_{j}^{\beta}$ and is therefore a join of CJP elements by the induction hypothesis. Therefore, $c \wedge b_{j}^{\beta}$ is a join of CJP elements.

We have established that $\boldsymbol{L}$ is bialgebraic. Therefore, $\boldsymbol{L}$ is isomorphic to $\mathbf{O}(\boldsymbol{R})$, where $\boldsymbol{R}=\mathbf{C J P}(\boldsymbol{L})$. We now prove that incomparable CJP elements of $\boldsymbol{L}$ are orthogonal. In view of Corollary 1.4 , this will suffice to prove that $L$ is a member of the class $\mathscr{I R \mathscr { N }}$.

Let $j, k$ be incomparable members of $\mathbf{C J P}(\boldsymbol{L})$. There exist least ordinals $\beta_{j}$ and $\beta_{k}$ such that $j \leq z^{\beta_{j}}$ and $k \leq z^{\beta_{k}}$. Let $\beta=\max \left\{\beta_{j}, \beta_{k}\right\}$. We may assume that $\beta=\beta_{j}$. Under this assumption, there exist unique $b_{1}^{\beta}$ and $b_{2}^{\beta}$ in $\mathbf{B}(\beta)$ such that $j \leq b_{1}^{\beta}, k \leq b_{2}^{\beta}$, and $b_{1}^{\beta} \notin \mathbf{B}(\xi)$ for all $\xi<\beta$. Since $\mathbf{B}(\boldsymbol{\beta})$ is an orthogonal set, it will suffice to prove that $b_{1}^{\beta} \neq b_{2}^{\beta}$.

By assumption, $b_{1}^{\beta}$ is a proper ordinal extension of the join of a set $\boldsymbol{D}$, where $D$ is constructed in one of two ways: If $\beta$ is a successor ordinal, then $D$ is a subset of $\mathbf{B}(\beta-1)$ containing at least two elements; if $\beta$ is a limit ordinal (possibly 0), then $\boldsymbol{D} \subseteq \bigcup\{\mathbf{B}(\xi): \xi<\beta\}$ is a chain such that $\boldsymbol{D} \cap \mathbf{B}(\xi) \neq \varnothing$ for all $\xi<\beta$. In either event, we must have $\bigvee \boldsymbol{D}<j \leq b_{1}^{\beta}$. In particular, $b_{1}^{\beta}$ is an ordinal extension of $j$. Consequently, $j$ is a node of $\downarrow b_{j}^{\beta}$; and it follows at once that $b_{1}^{\beta} \neq b_{2}^{\beta}$ (otherwise, $j$ would be comparable to $k$ ).

We have proven $\boldsymbol{L}$ to be a bialgebraic member of the class $\mathscr{I R \mathscr { N }}$. It remains to show that $\mathbf{M}(\boldsymbol{L})$ satisfies DCC. We prove that any descending chain in $\mathbf{M}(\boldsymbol{L})$ induces a descending chain in $\left\{z^{\beta}: \beta<\delta\right\}$. Suppose $g_{1}, g_{2} \in \mathbf{M}(\boldsymbol{L})$ and 
$g_{1}<g_{2}$. By Corollary 2.5, there exist CJP elements $j, k$ such that $g_{1}=j^{* *}$ and $g_{2}=k^{* *}$. Let $\xi, \eta<\delta$ be the least ordinals such that $j \leq b^{\xi}$ and $k \leq b^{\eta}$ for some $b^{\xi} \in \mathbf{B}(\xi)$ and $b^{\eta} \in \mathbf{B}(\eta)$. By Lemma 4.8, $j \leq b^{\xi} \leq g_{1}$ and $k \leq b^{\eta} \leq g_{2}$. Since $k$ is a node of $\downarrow g_{2}, g_{1}$ is comparable to $k$. As $g_{1}$ admits no proper ordinal extension, it follows that $g_{1}<k$. Hence, $b^{\xi}<b^{\eta}$, which implies $z^{\xi}<z^{\eta}$. Thus, any descending chain $\boldsymbol{C}$ in $\mathbf{M}(\boldsymbol{L})$ induces a descending chain in $\left\{z^{\beta}: \beta<\delta\right\}$ order-isomorphic to $\boldsymbol{C}$. Therefore, $\mathbf{M}(\boldsymbol{L})$ must satisfy DCC.

We now state and prove the main result of this paper.

Theorem 4.11. For an algebraic, distributive lattice $L$, the following are equivalent:

(1) The top element of $\boldsymbol{L}$ admits an ordinal decomposition over an orthogonal set of proper linear elements.

(2) $\boldsymbol{L}$ is isomorphic to $\mathbf{O}(\boldsymbol{R})$ for some root-system $\boldsymbol{R}$ in which every filet configuration is finite.

(3) $\boldsymbol{L}$ is a member of the class $\mathscr{G R N}$ and $\operatorname{Com}(\boldsymbol{L}) / \Theta$ satisfies $D C C$.

Proof. The equivalence of statements (2) and (3) was established in Corollary 3.4. Lemma 4.10, Corollary 1.4, and Theorem 3.3 together show that statement (1) implies statement (2). In what follows, we prove that statement (2) implies statement (1).

Suppose condition (2) is satisfied. We know by Corollary 3.5 that $\boldsymbol{L}$ has a basis $\mathbf{B}(0)$ which we may assume consists of maximal linear elements. We use Construction 4.7 to obtain an ordinal decomposition in $L$ over the set $\mathbf{B}(0)$. Let $0<\beta$ be an ordinal and consider the level $\mathbf{B}(\beta)$ of this decomposition. We assume $z^{\beta}<\mathrm{T}$ and prove the level $\mathbf{B}(\beta+1)$ can be constructed such that $z^{\beta}<z^{\beta+1}$.

In what follows, we shall use without explicit reference the fact that each level $\mathbf{B}(\xi), 0 \leq \xi \leq \beta$, is a maximal orthogonal set and a subset of $\mathbf{C M}(\boldsymbol{L})$.

Since $\beta+1$ is a successor ordinal, it will suffice to prove the existence of an element $b^{\beta+1}$ in $L$ which is the maximal ordinal extension of the join of a set $\boldsymbol{D} \subseteq \mathbf{B}(\beta)$ containing at least two elements.

Claim 1. There exists a CJP element $j$ and an element $b^{\beta} \in \mathbf{B}(\beta)$ such that $b^{\beta}<j$; and, for every pair of orthogonal CJP elements $c, d<j$, there exist minimal ordinals $\xi, \eta \leq \beta$ such that $c^{* *} \in \mathbf{B}(\xi)$ and $d^{* *} \in \mathbf{B}(\eta)$.

To prove this claim, we first show that whenever $j$ is a CJP element and $j \not z^{\beta}$, then there exists $b_{j}^{\beta} \in \mathbf{B}(\beta)$ such that $b_{j}^{\beta}<j$.

Suppose $j$ is a CJP element such that $j \not \leq z^{\beta}$. Since $\mathbf{B}(\beta)$ is a maximal orthogonal set and $j^{* *} \notin \mathbf{B}(\beta)$ by assumption, there exist $b_{j}^{\beta} \in \mathbf{B}(\beta)$ such that $\perp<j^{* *} \wedge b_{j}^{\beta}$. Since $j^{* *}, b_{j}^{\beta} \in \mathbf{C M}(\boldsymbol{L})$, it follows by Lemma 4.2 that $j^{* *}$ is comparable to $b_{j}^{\beta}$. Since $j \not z^{\beta}$, we see that $b_{j}^{\beta}<j \leq j^{* *}$.

We now prove the existence of a CJP element $j \not \leq z^{\beta}$ such that every pair $c, d$ of incomparable CJP elements below $j$ has the property that $c \vee d \leq z^{\beta}$.

Since $\boldsymbol{L}$ is bialgebraic and $z^{\beta}<\mathrm{T}$, there exists a CJP element $k \not z^{\beta}$. Suppose by way of contradiction that for every CJP element $j \leq k$ such that $j \not \leq z^{\beta}$ there exists a pair $c, d$ of incomparable CJP elements below $j$ with the property that either $c \not z^{\beta}$ or $d \not z^{\beta}$. 
By previous arguments, there exist $b_{k}^{\beta} \in \mathbf{B}(\beta)$ such that $b_{k}^{\beta}<k$. By construction, $b_{k}^{\beta}$ admits no proper ordinal extension; hence, $\downarrow k \backslash \downarrow b_{k}^{\beta}$ is not a chain. Consequently, there exist incomparable elements $c_{1}, c_{2} \in \downarrow k \backslash \downarrow b_{k}^{\beta}$, which we may assume are CJP since $\boldsymbol{L}$ is bialgebraic. Observe that $\boldsymbol{F}_{1}=$ $\left\{k, c_{1}\right\} \cup\left\{c_{2}\right\}$ and $\boldsymbol{G}_{1}=\left\{k, c_{2}\right\} \cup\left\{c_{1}\right\}$ are filet configurations in $\mathbf{C J P}(\boldsymbol{L})$.

We may assume that $c_{1} \notin z^{\beta}$. The previous arguments, therefore, may be repeated with $c_{1}$ in place of $k$. An inductive argument thus establishes the existence of an infinite filet configuration in $\operatorname{CJP}(L)$, contradicting Theorem 3.3.

Consequently, there exists a CJP element $j \not \leq z^{\beta}$ with the property that, whenever $c, d<j$ are incomparable CJP elements, then $c \vee d \leq z^{\beta}$. Hence, by Corollary 4.9, there exist least ordinals $\xi, \eta<\delta$ such that $c^{* *} \in \mathbf{B}(\xi)$ and $d^{* *} \in \mathbf{B}(\eta)$, completing the proof of Claim 1 .

Let $j$ and $b^{\beta}$ be as in Claim 1. We prove that $j^{* *}$ is the desired element $b^{\beta+1}$. To begin, let $\boldsymbol{D}=\{b \in \mathbf{B}(\beta): b<j\}$.

We show that $\boldsymbol{D}$ contains at least two elements. By Claim $1, j$ exceeds the element $b^{\beta} \in \mathbf{B}(\beta)$; hence, $\boldsymbol{D} \neq \varnothing$. By construction, $b^{\beta}$ admits no proper ordinal extension. Thus, there exist $h<j$ such that $h \| b^{\beta}$. Consequently, there exist orthogonal CJP elements $c \leq h$ and $d \leq b^{\beta}$. We may assume $c \| b^{\beta}$. By Claim 1, there exists a minimal ordinal $\xi \leq \beta$ such that $c^{* *} \in \mathbf{B}(\xi)$. By construction, there exist $b \in \mathbf{B}(\beta)$ such that $c^{* *} \leq b$.

Either $b=b^{\beta}$, or $b \| b^{\beta}$. If $b=b^{\beta}$, then $c \leq b^{\beta}$, contrary to assumption. Since $\perp<c \leq b \wedge j^{* *}$, it follows by Lemma 4.2 that $b$ is comparable to $j^{* *}$. Since $j \not z^{\beta}$, it follows that $b<j^{* *}$. Since $j$ is a node of $\downarrow j^{* *}$ and $b \| b^{\beta}$, it follows that $b<j$. Hence, $b \in \boldsymbol{D}$; and $\boldsymbol{D}$ contains at least two elements.

We now show an element $b \in \mathbf{B}(\beta)$ is in the set $\boldsymbol{D}$ if and only if $b$ is comparable to $j^{* *}$. It is clear that if $b \in \boldsymbol{D}$, then $b$ is comparable to $j^{* *}$. Let $b \in \mathbf{B}(\beta)$ be comparable to $j^{* *}$. We must have $b<j^{* *}$ since $j \not \leq z^{\beta}=\bigvee \mathbf{B}(\beta)$. Since $\downarrow j^{* *} \backslash \downarrow j$ is a chain and $b$ admits no proper ordinal extension, it follows that $b<j$. Hence, $b \in \boldsymbol{D}$.

Let $\boldsymbol{E}=\mathbf{B}(\beta) \backslash \boldsymbol{D}$. It follows that no element of $\boldsymbol{E}$ is comparable to $j^{* *}$; hence, by Lemma 4.2, $\boldsymbol{E}=\left\{b \in \mathbf{B}(\beta): b \wedge j^{* *}=\perp\right\}$.

In light of Lemma 2.4, to complete the proof that $j^{* *}$ is the desired element $b^{\beta+1}$, it will suffice to show that $j^{* *}$ is a proper ordinal extension of $\vee D$. Clearly, $\bigvee D<j \leq j^{* *}$; thus, by Lemma 2.2 , it will be enough to show that every compact element in $\downarrow j^{* *} \backslash \bigvee \boldsymbol{D}$ is CJP in $\boldsymbol{L}$.

Let $c \leq j^{* *}$ be a compact element which is not CJP. We show that $c \leq \bigvee D$. Since $j^{* *} \in \operatorname{Ord}(j)$, we may assume by Lemma 2.2 that $c<j$. Since $L$ is bialgebraic, by Theorem 1.3 and Corollary 1.4 there exist pairwise orthogonal CJP elements $j_{1}, \ldots, j_{n}$ such that $c=j_{1} \vee \cdots \vee j_{n}$. By Claim $1, j_{k}^{* *} \leq z^{\beta}$ for $1 \leq k \leq n$. Thus, $j_{k} \leq z^{\beta}$; it follows that $j_{k} \leq \bigvee \boldsymbol{D}$ or $j_{k} \leq \bigvee \boldsymbol{E}$. If $j_{k} \leq \bigvee \boldsymbol{E}$, then by JID and the description of $\boldsymbol{E}$ given above, we have

$$
j_{k}=j_{k} \wedge \bigvee \boldsymbol{E} \leq \bigvee\left\{j^{* *} \wedge b: b \in \boldsymbol{E}\right\}=\perp,
$$

contradicting the choice of $j_{k}$. Consequently, $j_{k} \leq \bigvee \boldsymbol{D}$ for all $1 \leq k \leq n$, proving that $c \leq \bigvee D$.

We have remarked that $\mathbf{D}$ is a member of the class $\mathscr{R N}$ if and only if I(D) is a member of the class $\mathscr{S R \mathscr { N }}$. In view of this fact, Theorem B is a 
restatement of Theorem 4.11 in terms of members of the class $\mathscr{R} \mathscr{N}$. Hence, the proof of Theorem B is complete.

Theorem 4.11 implies a result concerning posets:

If $\boldsymbol{P}$ is a poset, then the top element, $\boldsymbol{P}$, of $\mathbf{O}(\boldsymbol{P})$ admits an ordinal decomposition over an orthogonal set of proper linear elemenis of $\mathbf{O}(\boldsymbol{P})$ if and only if $\boldsymbol{P}$ is a root-system in which every filet configuration is finite.

The linear elements of $\mathbf{O}(\boldsymbol{P})$ are precisely those lower sets of $\boldsymbol{P}$ which are also chains; consequently, Theorem $\mathrm{C}$ is simply a restatement of the above in terms of the poset $\boldsymbol{P}$. It is important to note, however, that Theorem $\mathrm{C}$ does not imply Theorem 4.11. Indeed, Theorem 4.11 establishes that the class of algebraic, distributive lattices whose top elements admit ordinal decompositions over orthogonal sets of proper linear elements is precisely the class whose members are isomorphic to $\mathbf{O}(\boldsymbol{R})$ for some root-system $\boldsymbol{R}$ in which every filet configuration is finite. Theorem 4.11 is therefore stronger than Theorem $C$.

The proof of Theorem 4.11 makes no explicit use of those elements of $\mathbf{B}(\beta)$ which are trivial ordinal extensions of transfinite sequences. The reader may therefore be led to question the inclusion of such elements in the general definition of an ordinal decomposition. In order to show these elements cannot be omitted from the definition, we conclude this section by presenting the following example of a lattice whose top element admits an ordinal decomposition over an orthogonal set of linear elements requiring $\omega+1$ levels in which every element of $\mathbf{B}(\omega)$ is the trivial ordinal extension of a transfinite sequence.

Example 4.12. Let $\mathbf{A}=\left\{a_{N}: n<\omega\right\}$ be a countably infinite antichain and let $\mathbf{J}=\left\{j_{n}: 0<n<\omega\right\}$ be a countably infinite set disjoint from $\mathbf{A}$. Let $\mathbf{I}_{1}=\left\{a_{0}, a_{1}\right\} \oplus\left\{j_{1}\right\}$ and for all $n>1$, let $\mathbf{I}_{n}=\left(\mathbf{I}_{n-1} \cup\left\{a_{n}\right\}\right) \oplus\left\{j_{n}\right\}$. Let $\mathbf{R}=\bigcup\left\{\mathbf{I}_{n}: 1 \leq n<\omega\right\}$ and let $\mathbf{R}^{\prime}$ be an isomorphic copy of $\mathbf{R}$ with $\mathbf{R} \cap \mathbf{R}^{\prime}=\varnothing$. Let $\mathbf{S}=\left(\mathbf{R} \cup \overline{\mathbf{R}}^{\prime}\right) \oplus\{j\}$, where $j \notin \mathbf{R} \cup \mathbf{R}^{\prime}$. Finally, let $\boldsymbol{L}=\mathbf{O}(\mathbf{R})$ and $\mathbf{M}=\mathbf{O}(\mathbf{S})$. It is easy to check that the top element of $\mathbf{M}$ admits an ordinal decomposition over an orthogonal set of linear elements requiring $\omega+1$ levels. Note also that every element of $\mathbf{B}(\omega)$ is the join of a countable ascending chain.

\section{ADDITIONAL DECOMPOSITION THEOREMS}

For the remainder of this paper, when we refer to a decomposition, it will be understood that we mean an ordinal decomposition. We shall attach special importance to ordinal decompositions over orthogonal sets of proper linear elements. We will refer to such decompositions as linear-based decompositions.

Theorem 4.11 presents general conditions equivalent to the condition that the top element of an algebraic, distributive lattice admits a linear-based decomposition. In this section, we shall establish conditions which give greater control over these decompositions. We begin, however, by observing that specific questions about the structure of linear-based decompositions cannot be readily answered by appealing directly to Definition 4.1 as this construction does not allow sufficient control over the placement of elements within the $\beta$ levels. Precise control over the placement of elements is given by the canonical example of an ordinal decomposition given in Construction 4.7. In all that follows, we shall use the term canonical decomposition when referring to an ordinal decomposition whose levels are as described in Construction 4.7 and shall restrict attention, unless otherwise stated, to canonical decompositions. 
When reading this section, the reader will no doubt observe that Theorems 5.3-5.6 and 5.8-5.10 assume only algebraic, distributive lattices but draw conclusions concerning canonical decompositions of these lattices. Since canonical decompositions are defined only in members of the class $\mathscr{I R \mathscr { N }}$, the reader may question the validity of the conclusions. However, in view of Theorem 4.11 , the difficulty reduces to a question of semantics. If the top element of an algebraic, distributive lattice admits a linear-based decomposition, then it is

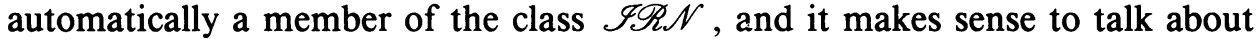
canonical decompositions in such a lattice. In view of this, we remark that in these theorems, we make the tacit assumption that the lattices under scrutiny satisfy the conditions of Theorem 4.11 .

The remainder of this section will be concerned with three successive refinements of Definition 4.1.

We say an element $g$ of a complete lattice $\boldsymbol{L}$ admits an $\omega$-decomposition if $g=\bigvee\left\{z^{n}: n<N\right\}, N \leq \omega$, the elements $z^{n}=\bigvee \mathbf{B}(n)$ are constructed as in Definition 4.1, and $z^{n}<z^{n+1}, n<N$. If $g$ admits an $\omega$-decomposition, and if for each element $b^{n+1} \in \mathbf{B}(n+1), b^{n+1} \wedge b=\perp$ for all but a finite number of elements $b \in \mathbf{B}(n)$, then we say $g$ admits a strong $\omega$-decomposition. If $g$ admits a strong $\omega$-decomposition over a finite set, then we say $g$ admits a finite decomposition.

Before exploring the refinements of Definition 4.1, we introduce some additional terminology and preliminary results.

A chain of $N+1$ distinct elements is said to have length $N$. Let $\boldsymbol{P}$ be a poset satisfying DCC. The height of an element $p \in \boldsymbol{P}$ is the supremum of the lengths of the descending chains in $\boldsymbol{P}$ with top element $p$. We shall denote the height of $p$ by $h_{P}(p)$, or simply by $h(p)$ when no confusion will result. It is clear that $h_{P}(p)$ is an ordinal not exceeding $\omega$. The height $h_{P}$ of a poset $\boldsymbol{P}$ satisfying DCC is the supremum of the heights of its elements.

Similarly, the length of a finite filet configuration $\boldsymbol{F}=\left\{a_{n}: 0 \leq n \leq N\right\} \cup$ $\left\{b_{n}: 1 \leq n \leq N\right\}$ is the number $N$; i.e., the length of the chain $\left\{a_{n}: 0 \leq\right.$ $n \leq N\}$. If $\boldsymbol{Q}$ is a poset in which every filet configuration is finite, we shall define the filet-height, $H_{Q}(q)$, or simply $H(q)$, of an element $q \in \boldsymbol{Q}$ to be the supremum of the lengths of all filet configurations in $\boldsymbol{Q}$ with top element $q$. The filet-height $H_{Q}$ of a poset $\boldsymbol{Q}$ in which every filet configuration is finite is the supremum of the filet heights of its elements.

The following lemma catalogues some simple observations about root-systems which will be used in later results.

Lemma 5.1. If $\boldsymbol{R}$ is a root-system, then each of the first three statements below implies the next:

(1) $\boldsymbol{R}$ has at most finitely many roots.

(2) $\boldsymbol{R}$ has finite filet height.

(3) Each element of $\boldsymbol{R}$ has finite filet height.

(4) Every filet configuration in $\boldsymbol{R}$ is finite.

Proof. We need only prove that (1) implies (2); the remaining implications are clear. Suppose $\boldsymbol{R}$ is a root-system containing exactly $N$ roots (maximal chains). For a proof by contradiction, suppose $N-1<H_{R}$. Then there exists an element $r \in \boldsymbol{R}$ such that $N \leq H(r)$; hence, there exists a filet configuration with top element $r$ of length $N$ in the principal lower set $\downarrow r$. It follows that 
$\downarrow r$ contains at least $N+1$ distinct roots. Zorn's Lemma implies that every root of $\downarrow r$ is contained in a root of $\boldsymbol{R}$. It is clear that two distinct roots of $\downarrow r$ cannot be contained in the same root of $\boldsymbol{R}$. Hence, $\boldsymbol{R}$ contains at least $N+1$ roots, contrary to assumption.

We remark in passing that the statements of Lemma 5.1 are not equivalent. For example, the lattice $\boldsymbol{M}$ constructed in Example 4.12 has the property that every filet configuration in $\mathbf{C J P}(\boldsymbol{M})$ is finite, yet $\mathbf{C J P}(\boldsymbol{M})$ has infinitely many roots.

The following result is an easy consequence of Corollary 2.5 and Lemma 3.1.

Lemma 5.2. Let $\boldsymbol{L}$ be a bialgebraic member of the class $\mathscr{I R N}$. A chain in $\mathbf{M}(\boldsymbol{L})$ has length $N$ if and only if it induces a filet configuration of length $N$ in $\operatorname{CJP}(L)$.

Lemma 5.3. Suppose $\boldsymbol{L}$ is an algebraic, distributive lattice whose top element admits a canonical, linear-based, $\omega$-decomposition. If $b^{n} \in \mathbf{B}(n) \quad(n<\omega)$ and $b^{n} \notin \mathbf{B}(k)$ for $0 \leq k<n$, then $b^{n}$ has height $n$ in $\mathbf{M}(\boldsymbol{L})$.

Proof. By Theorem 4.11, $L$ is isomorphic to $\mathbf{O}(\boldsymbol{R})$ for some root-system $\boldsymbol{R}$ in which every filet configuration is finite. We accomplish this proof by induction. Clearly the result is true if $n=0$. Let $n<\omega$ and suppose that, for all $0 \leq k<n$, if $b^{k} \in \mathbf{B}(k)$ and $b^{k} \notin \mathbf{B}(j)$ for $0 \leq j<k$, then $h\left(b^{k}\right)=k$ in $\mathbf{M}(\boldsymbol{L})$.

Let $b^{n} \in \mathbf{B}(n)$ be such that $b^{n} \notin \mathbf{B}(k)$ for $0 \leq k<n$. It follows that $b^{n}$ is a proper (indeed maximal) ordinal extension of $\bigvee \boldsymbol{D}$ for some $\boldsymbol{D} \subseteq \mathbf{B}(n-1)$ containing at least two elements. If $b \in \boldsymbol{B}(n-2)$, then $b \in \mathbf{B}(n-1)$ if and only if $[b]_{\sim_{B(n-2)}}=\{b\}$ (see Construction 4.7). Consequently, if every $b \in \boldsymbol{D}$ is contained in $\mathbf{B}(n-2)$, we would have $b^{n} \in \mathbf{B}(n-1)$, contrary to assumption. Thus, there must exist a $b^{n-1} \in \mathbf{D}$ such that $b^{n-1} \notin \mathbf{B}(k)$ for $0 \leq k<n-1$. By the induction hypothesis, $b^{n-1}$ has height $n-1$ in $\mathbf{M}(\boldsymbol{L})$. Thus, there exists a strictly ascending chain of maximal ordinal elements $C_{n-1}\left(b^{n-1}\right)=\left\{b^{0}, b^{1}, \ldots, b^{n-1}\right\}$ such that $b^{j} \in \mathbf{B}(j)$ for each $0 \leq j \leq n-1$. The chain $C_{n}\left(b^{n}\right)=C_{n-1}\left(b^{n-1}\right) \cup\left\{b^{n}\right\}$ contains exactly $n+1$ elements. Hence, $n \leq h\left(b^{n}\right)$ in $\mathbf{M}(\boldsymbol{L})$.

If $\boldsymbol{C}$ is any chain of maximal ordinal elements contained in $\downarrow b^{n}$ and $c \in C$, then by Corollary 4.9, $c \in \bigcup\{\mathbf{B}(j): j \leq n\}$. It follows that $C$ has length at most $n$.

Theorem 5.4. For an algebraic, distributive lattice $L$, the following are equivalent:

(1) The top element of $\boldsymbol{L}$ admits a canonical, linear-based, $\omega$-decomposition.

(2) $\boldsymbol{L}$ is a bialgebraic (finite-valued) member of the class $\mathscr{I R R}$, and every maximal ordinal element of $\boldsymbol{L}$ has finite height in $\mathbf{M}(\boldsymbol{L})$.

(3) $\boldsymbol{L}$ is isomorphic to $\mathbf{O}(\boldsymbol{R})$ for some root-system $\boldsymbol{R}$ in which every element has finite filet-height.

Proof. The equivalence of conditions (2) and (3) follows directly from Theorem 3.3, Corollary 3.4, and Lemma 5.2. In what follows, we prove that (1) implies (2), and (3) implies (1).

(1) $\Rightarrow(2)$ Let $\mathrm{T}=\bigvee\left\{z^{n}: n<N\right\} \quad(N \leq \omega)$, where each $z^{n}=\bigvee \mathbf{B}(n)$, each set $\mathbf{B}(n) \subseteq \mathbf{C M}(\boldsymbol{L})$ is as described in Construction 4.7, and where $\mathbf{B}(0)$ is an 
orthogonal set of maximal linear elements. By Theorem 4.11, we know $L$ is isomorphic to $\mathbf{O}(\boldsymbol{R})$ for some root-system $\boldsymbol{R}$ (in which every filet configuration is finite) and is thus bialgebraic by Corollary 3.4 .

If $g \in \mathbf{M}(\boldsymbol{L})$, then there is a least integer $k$ such that $g \in \mathbf{B}(k)$ by Corollary 4.9. By Lemma 5.3, $h(g)=k$ in $\mathbf{M}(L)$; hence, every maximal ordinal element in $\boldsymbol{L}$ has finite height in $\mathbf{M}(\boldsymbol{L})$.

$(3) \Rightarrow(1)$ In view of Lemma 5.1, it is clear that every filet configuration in $\boldsymbol{R}$ is finite. By Theoren 4.11, the top element of $\boldsymbol{L}$ admits a canonical, linearbased decomposition. Suppose by way of contradiction that the level $\mathbf{B}(\omega+1)$ of this decomposition is such that $z^{\beta}<z^{\omega+1}$ for all $\beta \leq \omega$.

There exists an element $b^{\omega+1} \in \mathbf{B}(\omega+1)$ which is not contained in $\mathbf{B}(\beta)$ for any $\beta \leq \omega$. Hence, there exists a set $D \subseteq \mathbf{B}(\omega)$ containing at least two elements such that $b^{\omega+1}$ is a proper (indeed maximal) ordinal extension of $\checkmark D$.

Suppose that $\boldsymbol{D} \subseteq \bigcup\{\mathbf{B}(n): n<\omega\}$. If there exists an integer $0 \leq k$ such that $\boldsymbol{D} \subseteq \bigcup\{\mathbf{B}(n): n \leq k\}$, then by Construction $4.7, b^{\omega+1} \in \mathbf{B}(k+1)$, contrary to assumption. Thus, for each integer $n<\omega$, there exists a $b \in \boldsymbol{D}$ and a least integer $k>n$ such that $b \in \mathbf{B}(k)$. By Construction 4.7, $b \in \mathbf{M}(\boldsymbol{L})$; by Lemma 5.3, $h(b)=k$. Since $b<b^{\omega+1}$ for all $b \in \boldsymbol{D}$, it follows that $h\left(b^{\omega+1}\right)=\omega$ in $\mathbf{M}(\boldsymbol{L})$, contradicting condition (2).

Suppose instead that $\boldsymbol{D} \nsubseteq \bigcup\{\mathbf{B}(n): n<\omega\}$. Then there exists an element $b^{\omega} \in \boldsymbol{D}$ such that $b^{\omega} \notin \mathbf{B}(n)$ for all $n<\omega$. By construction, $b^{\omega} \in \operatorname{Ord}(\bigvee \boldsymbol{W})$ for some chain $\boldsymbol{W} \subseteq \bigcup\{\mathbf{B}(n): n<\omega\}$ such that $\boldsymbol{W} \cap \mathbf{B}(n) \neq \varnothing$ for all $n<\omega$. Let $n<\omega$. By assumption, there exists $w \in \boldsymbol{W}$ such that $w \leq b^{n}$ for all $b^{r} \in \mathbf{B}(n)$. Let $k>n$ be the least integer such that $w \in \mathbf{B}(k)$. By Construction 4.7, $w \in \mathbf{M}(\boldsymbol{L})$; and, by Lemma 5.3, $h(w)=k$ in $\mathbf{M}(\boldsymbol{L})$. Thus, for each integer $n<\omega$, there exists an element $w \in \boldsymbol{W}$ such that $h(w)>n$ in $\mathbf{M}(\boldsymbol{L})$. Since each $w<b^{\omega+1}$, it follows that $h\left(b^{\omega+1}\right)=\omega$ in $\mathbf{M}(\boldsymbol{L})$, contradicting condition (2).

Note that the top element of the lattice R:1 introduced in Example 4.12 does not admit a canonical, linear-based $\omega$-decomposition. However, the top element of the lattice $\boldsymbol{L}$ in this example does admit such a decomposition; in fact, it admits a canonical, linear-based strong $\omega$-decomposition.

Suppose an element $g$ of a complete lattice $\boldsymbol{L}$ admits an ordinal decomposition as described in Definition 4.1. We will say the ascending chain $\left\{z^{\beta}: \beta<\delta\right\}$ terminates at $\alpha$ provided there is an ordinal $\alpha<\delta$ such that $z^{\alpha}=g$ and $\bigvee\left\{z^{\beta}: \beta<\alpha\right\}<g$.

The top element of the lattice $M$ of Example 4.12 admits a canonical, linearbased decomposition which terminates at the ordinal $\omega+1$. On the other hand, the top element of the lattice $\boldsymbol{L}$ of Example 4.12 admits a canonical, linearbased, strong $\omega$-decomposition which does not terminate. Our next goal will be to characterize those algebraic, distributive lattices whose top elements admit canonical, linear-based decompositions which terminate at some integer $N$.

Theorem 5.5. Let $\boldsymbol{L}$ be an algebraic, distributive lattice and let $N$ be an integer. The following are equivalent:

(1) The top element of $\boldsymbol{L}$ admits a canonical, linear-based decomposition which terminates at $N$. 
(2) $\boldsymbol{L}$ is a bialgebraic member of the class $\mathscr{G R N}$ and $\mathbf{M}(\boldsymbol{L})$ has height $N$.

(3) $\boldsymbol{L}$ is isomorphic to $\mathbf{O}(\boldsymbol{R})$ for some root-system $\boldsymbol{R}$ having filet-height $N$.

Proof. The equivalence of conditions (2) and (3) is a direct consequence of Corollary 3.4 and Lemma 5.2. We prove the equivalence of conditions (1) and (2).

(1) $\Rightarrow$ (2) Suppose that $T=z^{0} \vee z^{1} \vee \cdots \vee z^{N}$, where $z^{j}=\vee \mathbf{B}(j)$, the sets $\mathbf{B}(j)$ are as described in Construction 4.7 for $0 \leq j \leq N$, and $\mathbf{B}(0)$ is a maximal orthogonal set of maximal linear elements.

By Theorem 4.11, $\boldsymbol{L}$ is isomorphic to $\mathbf{O}(\boldsymbol{R})$ for some root-system (in which every filet configuration is finite) and is therefore a bialgebraic member of the class $\mathscr{S R N}$ by Corollary 3.4. Since $z^{0} \vee \cdots \vee z^{N-1}<\mathrm{T}$, there exists a $b^{N} \in$ $\mathbf{B}(N)$ which is not contained in $\mathbf{B}(j)$ for all $0 \leq j<N$. By Lemma 5.3, $h\left(b^{N}\right)=N$ in $\mathbf{M}(\boldsymbol{L})$. Thus, $N \leq h_{M(L)}$.

Let $C$ be any descending chain of maximal ordinal elements in $L$. By Corollary 4.9, each member of $\boldsymbol{C}$ must be contained in $\mathbf{B}(j)$ for some $0 \leq$ $j \leq N$. It follows that $C$ has length at most $N$. Thus, $h_{M(L)} \leq N$.

$(2) \Rightarrow(1)$ Clearly, $\mathbf{M}(\boldsymbol{L})$ satisfies DCC. Since $\boldsymbol{L}$ is a bialgebraic member of the class $\mathscr{I R N}, L$ is finite-valued by Corollary 1.4; thus, by Theorem 3.3, $\operatorname{Com}(\boldsymbol{L}) / \Theta$ satisfies DCC. Hence, $\boldsymbol{L}$ satisfies the conditions of Theorem 4.11; consequently, the top element of $\boldsymbol{L}$ admits a canonical, linear-based decomposition. If the level $\mathbf{B}(N+1)$ can be constructed such that $z^{N}<z^{N+1}$, then there must exist a $b^{N+1} \in \mathbf{B}(N+1)$ such that $b^{N+1} \notin \mathbf{B}(j)$ for $0 \leq j \leq N$. By Lemma 5.3, $h\left(b^{N+1}\right)=N+1$ in $\mathbf{M}(\boldsymbol{L})$. Therefore, we must have $N+1 \leq h_{M(L)}$, contradicting condition (2).

To see that the decomposition terminates at the integer $N$, it now suffices to show $\mathbf{B}(N)$ contains an element $b^{N}$ which is not contained in $\mathbf{B}(j)$ for $0 \leq j<N$. Let $\boldsymbol{C}$ be any chain of maximal ordinal elements in $\boldsymbol{L}$ of length $N$. By Corollary 4.9 , every member of $C$ must be contained in $\mathbf{B}(j)$ for some $0 \leq j \leq N$. It follows that $C \cap \mathbf{B}(j) \neq \varnothing$ for all $0 \leq j \leq N$. Consequently, the top element, $c$, of $\boldsymbol{C}$ is a member of $\mathbf{B}(N)$, and $c \notin \mathbf{B}(j)$ for $0 \leq j<N$.

Our next goal will be to characterize all members of the class $\mathscr{I R N}$ whose top elements admit canonical, linear-based strong $\omega$-decompositions. We begin by introducing some preliminary results.

Lemma 5.6. If $\boldsymbol{L}$ is an algebraic, distributive lattice whose top element admits a canonical, linear-based decomposition, then the first level of this decomposition forms a basis for $\boldsymbol{L}$.

Proof. By assumption, the first level, $\mathbf{B}(0)$, of this decomposition consists of pairwise orthogonal (maximal) linear elements. We need only show $\mathbf{B}(0)$ is a maximal orthogonal set. Since $\boldsymbol{L}$ is bialgebraic by Theorem 4.11, it will suffice to show that $j \in \mathbf{C J P}(\boldsymbol{L})$ implies $0<j \wedge b$ for some $b \in \mathbf{B}(0)$.

Let $j$ be CJP in $L$. There exists a least ordinal $\beta$ such that $j \leq z^{\beta}$. By Corollary 4.9, $j^{* *} \in \mathbf{B}(\beta)$. Consequently, there exist $b \in \mathbf{B}(0)$ such that $b \leq j^{* *}$. Since $j$ is a node of $\downarrow j^{* *}$, it follows that $\perp<j \wedge b$.

Lemma 5.7. If $\boldsymbol{L}$ is a member of the class $\mathscr{Y R N}$ and has a basis containing exactly $N$ elements, then every orthogonal set in $L \backslash\{\perp\}$ contains at most $N$ elements. 
Proof. The assumption implies (see remarks following Corollary 2.7) that the Boolean skeleton $\mathbf{B}(\boldsymbol{L})$ has $N$ atoms. If $\boldsymbol{D}$ is an orthogonal set in $\boldsymbol{L}$, then so is the set $\boldsymbol{D}^{\prime}=\left\{d^{* *}: d \in \boldsymbol{D}\right\} \subseteq \mathbf{B}(L)$. Since every element of $\boldsymbol{D}^{\prime}$ must exceed an atom of $\mathbf{B}(\boldsymbol{L})$, it follows that $\boldsymbol{D}^{\prime}$ contains at most $N$ elements. Thus, $\boldsymbol{D}$ contains at most $N$ elements.

Recall that a lower-bounded, distributive lattice $\boldsymbol{D}$ is orthofinite provided every orthogonal, upper-bounded subset of $D$ is finite. (See Lemmas 3.6 and 3.7.)

Theorem 5.8. For an algebraic, distributive lattice $\boldsymbol{L}$ the following are equivalent:

(1) The top element of $\boldsymbol{L}$ admits a canonical, linear-based, strong $\omega$-decomposition.

(2) $\boldsymbol{L}$ is a member of the class $\mathscr{G R N}$ and $\operatorname{Com}(\boldsymbol{L})$ is an orthofinite lattice.

(3) $\boldsymbol{L}$ is isomorphic to $\mathbf{O}(\boldsymbol{R})$ for some root-system $\boldsymbol{R}$ with the property that, for each $r \in \boldsymbol{R}$, the root-system $\downarrow r$ contains at most finitely many roots.

Proof. (1) $\Rightarrow(2)$ Suppose condition (1) is satisfied and let $c \in \operatorname{Com}(L)$. By Theorem $4.11, L$ is bialgebraic; hence, it will suffice to show that every orthogonal set $J$ of CJP elements contained in $\downarrow c$ is finite. By Theorem 1.3, $c$ has a unique representation as a finite join of pairwise orthogonal CJP elements, $c_{1}, \ldots, c_{m}$.

Let $\boldsymbol{J}$ be an orthogonal set of CJP elements contained in $\downarrow c$ and let $\boldsymbol{J}_{k}=$ $\left\{j \in \boldsymbol{J}: j \leq c_{k}\right\}$ for $1 \leq k \leq m$. It will suffice to show $\boldsymbol{J}_{k}$ is finite.

Let $\mathrm{T}=\bigvee\left\{z^{n}: n<N\right\} \quad(N \leq \omega)$, where each $z^{n}=\bigvee \mathbf{B}(n)$ is as described in Construction $4.7, \mathbf{B}(0)$ is an orthogonal set of proper (maximal) linear elements, and where each element of $\mathbf{B}(n+1)$ is orthogonal with all but a finite number of elements of $\mathbf{B}(n)$. Consider the CJP element $c_{k}, 1 \leq k \leq m$. Since $c_{k}^{* *} \in \mathbf{M}(\boldsymbol{L})$, there exists an integer $n$ such that $c_{k}^{* *} \in \mathbf{B}(n)$ by Corollary 4.9. Therefore, by construction, $c_{k}^{* *}$ admits a finite ordinal decomposition over a finite subset of $\mathbf{B}(0)$. By Lemma 5.6, the lattice $\downarrow c_{k}^{* *}$ has a finite basis. Consequently, $\boldsymbol{J}_{k}$ is finite by Lemma 5.7.

$(2) \Rightarrow(3)$ Suppose condition (2) is satisfied. Clearly every strong filet configuration in $\operatorname{Com}(\boldsymbol{L})$ must be finite. Consequently, $\boldsymbol{L}$ is bialgebraic by Theorem 3.3 and Corollary 3.4 and Corollary 3.2. By hypothesis, there are at most finitely many pairwise incomparable (hence orthogonal) CJP elements below $j$. It follows that the principal lower set $\downarrow j$ in $\mathbf{C J P}(\boldsymbol{L})$ contains at most finitely many roots.

(3) $\Rightarrow$ (1) Suppose condition (3) is satisfied. We see by Lemma 5.1 that $\boldsymbol{L}$ satisfies the conditions of Theorem 5.4. Hence, $T$ admits a canonical, linearbased $\omega$-decomposition. It remains to show that every element of $\mathbf{B}(n+1)$ is orthogonal with all but a finite number of elements of $\mathbf{B}(n)$.

Let $b \in \mathbf{B}(n+1)$. If $b \in \mathbf{B}(n)$, there is nothing to show. If $b \notin \mathbf{B}(n)$, then $b$ is a proper (indeed maximal) ordinal extension of $\vee \boldsymbol{D}$, where $\boldsymbol{D} \subseteq \mathbf{B}(n)$ contains at least two elements. By Lemma 4.5, $b \wedge b^{n}=\perp$ for all $b^{n} \in \mathbf{B}(n) \backslash D$; hence, it will suffice to show $D$ is finite. By Lemma 2.2 , there exists a CJP element $j$ such that $\bigvee D<j$. Since $D$ is an orthogonal set, it follows that $D$ must be finite.

Combining Theorems 5.5 and 5.8, we obtain the following: 
Theorem 5.9. For an algebraic distributive lattice $\boldsymbol{L}$ and an integer $N$, the following are equivalent:

(1) The top element of $\boldsymbol{L}$ admits a canonical, linear-based, strong $\omega$-decomposition which terminates at $N$.

(2) $L$ is a member of the class $\mathscr{G R N}$ and has the following properties:

(A) $\mathbf{M}(\boldsymbol{L})$ has height $N$.

(B) $\operatorname{Com}(\boldsymbol{L})$ is an orthofinite lattice.

(3) $\boldsymbol{L}$ is isomorphic to $\mathbf{O}(\boldsymbol{R})$ for some root-system $\boldsymbol{R}$ having the following properties:

(A) $\boldsymbol{R}$ has filet height $N$.

(B) For each $r \in \boldsymbol{R}$, the root-system $\downarrow r$ contains at most finitely many roots.

The following result characterizes those members of the class $\mathscr{A R N}$ whose top elements admit canonical, linear-based finite decompositions.

Theorem 5.10. Let $L$ be an algebraic, distributive lattice and let $N$ be any integer. The following are equivalent:

(1) The top element of $\boldsymbol{L}$ admits a canonical, linear-based, finite decomposition whose initial level contains exactly $N$ elements.

(2) $\boldsymbol{L}$ is a member of the class $\mathscr{I R N}$ such that every orthogonal subset of $\boldsymbol{L}$ contains at most $N$ elements, and there is one such set containing exactly $N$ elements.

(3) $\boldsymbol{L}$ is isomorphic to $\mathbf{O}(\boldsymbol{R})$ for some root-system containing exactly $N$ roots.

Proof. (1) $\Rightarrow(2) \quad L$ has a basis containing $N$ elements for some integer $N$ by Lemma 5.6. Every orthogonal subset of $\boldsymbol{L}$ must contain at most $N$ elements by Lemma 5.7 .

(2) $\Rightarrow$ (3) It is clear that if $c$ is compact in $L$, then every orthogonal subset of $\downarrow c$ must be finite. Hence, $L$ satisfies the conditions of Theorem 5.8 and is therefore isomorphic to $\mathbf{O}(\boldsymbol{R})$ for some root-system $\boldsymbol{R}$. Since $\boldsymbol{L}$ is bialgebraic and contains an orthogonal subset containing exactly $N$ elements, it follows that $\mathbf{C J P}(\boldsymbol{L})$ contains an orthogonal subset containing exactly $N$ elements. Hence, $\boldsymbol{R}$ contains at least $N$ distinct roots. If $\boldsymbol{R}$ contains $N+1$ roots, then there exists an orthogonal subset of $\operatorname{CJP}(\boldsymbol{L})$ containing $N+1$ elements, contradicting condition (2). Consequently, $\boldsymbol{R}$ contains exactly $N$ roots.

(3) $\Rightarrow$ (1) Clearly $\boldsymbol{L}$ satisfies the conditions of Theorem 5.9; it follows that the top element of $\boldsymbol{L}$ admits a canonical, linear-based strong $\omega$-decomposition which terminates at some integer $M$. It remains to show that $\mathbf{B}(0)$ contains exactly $N$ elements. By Corollary $3.5, \boldsymbol{L}$ has a basis $\boldsymbol{B}$, which we may assume consists of CJP elements. Since each member of $B$ must be contained in a root of $\mathbf{C J P}(\boldsymbol{L})$ and since $B$ is a maximal orthogoral subset of $L$, it follows that $\boldsymbol{B}$ contains exactly $N$ elements. Since $\boldsymbol{B}(0)$ is an orthogonal subset of $\boldsymbol{L}$, it follows by Lemma 5.7 that $\mathbf{B}(0)$ contains at most $N$ elements. In fact, since every element of $\mathbf{B}(0)$ must exceed a member of $\mathbf{B}$ by Corollary 2.7 and Lemma $2.4, \mathbf{B}(0)$ contains exactly $N$ elements.

It is well known that every infinite Boolean algebra contains an infinite orthogonal set. Thus, any Boolean algebra having the property that each of its 
orthogonal sets is finite must itself be finite. Consequently, in light of Corollary 2.7 and Lemma 5.6, a relatively normal lattice has the property that each of its orthogonal subsets is finite if and only if the lattice has a finite basis. Thus, Theorem $\mathrm{A}$, as stated in the introduction, is equivalent to Theorem 5.10.

A bounded, pseudocomplemented, distributive lattice $D$ is a Stone lattice provided $x^{*} \vee x^{* *}=\mathrm{T}$ for all $x \in D$. We conclude this section by using the decomposition theory developed thus far to characterize all bialgebraic Stone lattices in the class $\mathscr{G R} \mathscr{N}$. Before stating and proving this result, we summarize some relevant facts about the Boolean skeleton of an algebraic, distributive lattice.

Let $\boldsymbol{L}$ be an algebraic, distributive lattice and recall that the Boolean skeleton $\mathbf{B}(\boldsymbol{L})=\left\{a \in \boldsymbol{L}: a=a^{* *}\right\}$ of $\boldsymbol{L}$ forms a Boolean algebra $(\mathbf{B}(\boldsymbol{L}) ; \wedge, \sqcup, *, \perp, \mathrm{T})$, where the join operation is defined by $a \sqcup b=(a \vee b)^{* *}$. The Boolean algebra $\mathbf{B}(\boldsymbol{L})$, being the image of the closure operator $a \mapsto a^{* *}$ on $\boldsymbol{L}$, is a complete lattice and is completely meet-faithful in $L$; that is, arbitrary meets in $\mathbf{B}(\boldsymbol{L})$ coincide with the corresponding meets in $L$. However, $\mathbf{B}(\boldsymbol{L})$ is not, in general, a sublattice of $\boldsymbol{L}$.

Theorem 5.11. For a member $\boldsymbol{L}$ of the class $\mathscr{G R \mathscr { N }}$, the following are equivalent:

(1) $\boldsymbol{L}$ is bialgebraic and a Stone lattice.

(2) $\mathbf{B}(\boldsymbol{L})$ is atomic and completely join-faithful in $\boldsymbol{L}$.

(3) The top element of $\boldsymbol{L}$ is the join of an orthogonal set of maximal linear elements.

(4) $\boldsymbol{L}$ is bialgebraic, and $\mathbf{M}(\boldsymbol{L})$ is an antichain.

(5) $\boldsymbol{L}$ is isomorphic to $\mathbf{O}(\boldsymbol{R})$ for some root-system $\boldsymbol{R}$ consisting of a family of pairwise orthogonal chains.

Proof. The equivalence of conditions (3), (4), and (5) is a direct consequence of Theorem 5.5. It is also clear that (2) implies (1). In what follows, we prove that (1) implies (5), (3) implies (2), and (2) implies (3).

$(1) \Rightarrow(5)$ Suppose condition (1) is satisfied. We show that $\operatorname{CJP}(\boldsymbol{L})$ is a disjoint union of chains. Since $L$ is bialgebraic, it will suffice to show that $\downarrow j$ is a chain in $L$ for all $j \in \mathbf{C J P}(\boldsymbol{L})$.

To this end, let $j$ be CJP in $L$. Let $j_{1} \leq j$ and suppose $j_{2} \| j_{1}$ in $\boldsymbol{L}$. We show $j \| j_{2}$. We may assume $j_{1}$ and $j_{2}$ are CJP elements. By Lemma 1.2, $j_{1} \wedge j_{2}=\perp$; hence, $j_{1}^{* *} \wedge j_{2}^{* *}=\perp$ by Corollary 2.5 and Lemma 2.3. Since $\boldsymbol{L}$ is a Stone lattice, it now follows that $j_{1}^{*} \vee j_{2}^{*}=\mathrm{T}$. Thus, $j \leq j_{k}^{*}$ for some $k \in\{1,2\}$. If $j \leq j_{k}^{*}$, then $j \| j_{k}$. Since $j_{1} \leq j$, it now follows that $j \| j_{2}$.

(3) $\Rightarrow(2)$ Since the top element of $\boldsymbol{L}$ admits a canonical, linear-based ordinal decomposition, we know by Theorem 4.11 that $L$ is a bialgebraic member of the class $\mathscr{S R N}$. By Lemma 5.6, B(0) forms a basis of maximal linear elements for $\boldsymbol{L}$. Consequently, $\mathbf{B}(\boldsymbol{L})$ is atomic by Corollary 2.7; in particular, $\mathbf{B}(0)$ is the set of atoms for $\mathbf{B}(\boldsymbol{L})$.

Let $\boldsymbol{X} \subseteq \mathbf{B}(\boldsymbol{L})$. To see that $\mathbf{B}(\boldsymbol{L})$ is a complete sublattice of $\boldsymbol{L}$, we must show that $\bigsqcup \boldsymbol{X}=\bigvee \boldsymbol{X}$. By definition, $\bigsqcup \boldsymbol{X}=(\bigvee \boldsymbol{X})^{* *}$, and it is clear that $\bigvee \boldsymbol{X} \leq(\bigvee \boldsymbol{X})^{* * *}$. Since $\boldsymbol{L}$ is bialgebraic, to prove the reverse inequality, it will suffice to show that every CJP element below $(\bigvee X)^{* *}$ is also below $\bigvee \boldsymbol{X}$. Let $j \leq(\bigvee X)^{* *}$ be CJP in $\boldsymbol{L}$. By Corollary $4.9, j^{* *} \in \mathbf{B}(0)$ and is therefore an 
atom of $\mathbf{B}(\boldsymbol{L})$. If $j^{* *} \| x$ for all $x \in \boldsymbol{X}$, then by JID,

$$
j^{* *} \wedge \bigvee \boldsymbol{X}=\bigvee\left\{j^{* *} \wedge x: x \in \boldsymbol{X}\right\}=\perp
$$

This, however, implies $j^{* *} \leq(\bigvee X)^{*}$-an impossibility since $j^{* *} \leq(\bigvee X)^{* *}$. Consequently, $j \leq \bigvee \boldsymbol{X}$.

$(2) \Rightarrow(3)$ Since $\mathbf{B}(\boldsymbol{L})$ is a complete, atomic sublattice of $\boldsymbol{L}$, it follows that $\mathrm{T}=\bigvee \operatorname{At}(\mathbf{B}(L))$, where $\operatorname{At}(\mathbf{B}(L))$ denotes the set of atoms of $\mathbf{B}(\boldsymbol{L})$. Since $L$ is a member of the class $\mathscr{G R} \mathscr{N}$, it follows by Corollary 2.7 that every member of $\operatorname{At}(\mathbf{B}(L))$ is a maximal linear element of $\boldsymbol{L}$. Let $\mathbf{B}(0)=\mathbf{A t}(\mathbf{B}(\boldsymbol{L}))$.

We have seen that Theorem 4.11 can be restated as a result about members of the class $\mathscr{R} \mathscr{N}$ (see Theorem B in the introduction). Likewise, each of the decomposition theorems appearing in this section has a corresponding restatement in the context of relatively normal lattices. Furthermore, each of these theorems implies a decomposition theorem for posets in the spirit of Theorem C. These theorems are straightforward adaptations of Theorems B and C and are left to the reader.

\section{CONNECTIONS WITH ORDER-ALGEBRA}

In this section, we explore the connections between the theory of ordinal decompositions as developed in this paper and known results from the arena of order-algebra. We begin by reviewing some relevant concepts concerning lattice-ordered groups. For proofs and definitions not given here, the reader is referred to Anderson and Feil [2] or Bigard et al. [5].

A lattice-ordered group is an algebra $(G ;+,-, 0, \wedge, \vee)$ such that $(G ;+,-$, $0)$ is a (not necessarily abelian) group, $(G ; \wedge, \vee)$ is a lattice, and addition distributes over the lattice operations.

It is well known that the group reduct of $G$ is torsion free and the lattice reduct of $G$ is distributive. Furthermore, the equivalence " $a \leq b \Leftrightarrow 0 \leq a-b$ " shows that the order of $G$ is determined by the sublattice $G^{+}=\{g \in G: 0 \leq g\}$ of positive elements.

We shall adopt the usual abbreviations l-group for a lattice-ordered group, $l$-subgroup for a subalgebra of an $l$-group, and $l$-homomorphism for a homomorphism of $l$-groups. We also reserve the term $o$-group for an $l$-group whose lattice order is a total order. A convex l-subgroup of an $l$-group $G$ is an $l$ subgroup which is order-convex in $G$. An l-ideal of an $l$-group $G$ is a normal convex $l$-subgroup of $G$.

The algebraic closure families of convex $l$-subgroups and $l$-ideals of an $l$ group $G$ will be denoted by $\mathscr{C}(G)$ and $\mathscr{I}(G)$, respectively. The compact elements of $\mathscr{C}(G)$ (respectively, $\mathscr{I}(G)$ ) are the principal convex l-subgroups of $G$ (respectively, the principal $l$-ideals of $G$ ). We note that the $l$-ideals of $G$ are the kernels of $l$-homomorphisms with domain $G$; hence, $\mathscr{I}(G)$ is isomorphic to the congruence lattice of $G$.

It can be shown (see, for example, Anderson and Feil [2] or Bigard et al. [5]) that $\mathscr{C}(G)$ is always a member of the class $\mathscr{I R \mathscr { N }}$ and that the same is true for $\mathscr{I}(G)$ whenever $G$ is a subdirect product of $o$-groups.

The "Conrad Program" for studying $l$-groups involves analyzing the structure of individual $l$-groups (or classes of $l$-groups) by using only the strictly latticetheoretic properties of their lattices of convex $l$-subgroups. Conrad's papers in 
the 1960s pioneered this approach and amply demonstrated its usefulness. A survey of the most important consequences of this approach to l-groups can be found in Anderson et al. [1]; complete proofs for most of the surveyed results can be found in Conrad's "Blue Notes" [12].

The present study and its predecessors, Snodgrass and Tsinakis [26, 27], clarify the role of the lattice-theoretic component of $l$-group theory by demonstrating that the "Conrad Program" can be carried out in the more general setting of relatively normal lattices.

At this point, it is appropriate to consider the open question of whether every member of the class $\mathscr{I R \mathscr { N }}$ is isomorphic to the lattice of convex $l$-subgroups of an l-group. An affirmative answer for a significant special case is given by Theorem $\mathrm{D}$ (see the introduction). We may restate Theorem $\mathrm{D}$ in the language of the class $\mathscr{I R N}$ as follows:

For a member of the class $\mathscr{I R \mathscr { N }}$, the Glivenko quotient $\operatorname{Com}(\boldsymbol{L}) / \boldsymbol{\Theta}$ satisfies $D C C$ if and only if $\boldsymbol{L}$ is isomorphic to the lattice of convex l-subgroups (l-ideals) of an abelian l-group.

The proof of the nontrivial implication hinges upon the fact that $\boldsymbol{L}$ is bialgebraic (Theorem 3.3) and that every bialgebraic member of the class $\mathscr{I R \mathscr { N }}$ has the required representation (Conrad [10, Theorem 4.2]). We outline the method by which the appropriate $l$-group is chosen:

Let $\Lambda=\mathbf{C M I}(\boldsymbol{L})$. For each $\lambda \in \Lambda$, let $\mathbb{R}_{\lambda}$ be the $o$-group of real numbers. Let $G$ be the subgroup of the cartesian product $\Pi\left\{\mathbb{R}_{\lambda}: \lambda \in \Lambda\right\}$ consisting of all elements with finite support, and define an element of $G$ to be positive if and only if it is positive at each maximal element of its support. It is easily seen that $G$ is an abelian $l$-group with respect to this order; it is often called a restricted Hahn group and is denoted by $\sum\left(\Lambda, \mathbb{R}_{\lambda}\right)$. Conrad's aforementioned result asserts that $\boldsymbol{L}$ is isomorphic to the lattice of convex $l$-subgroups of $\sum\left(\Lambda, \mathbb{R}_{\lambda}\right)$.

An $l$-group $G$ is a lex-extension of $H \in \mathscr{C}(G)$ if $H$ is a prime (meetirreducible) element of $\mathscr{C}(G)$ and every element of $G^{+} \backslash H$ exceeds every element of $H$. It is easy to see that $G$ is a lex-extension of $H$ if and only if $G$ is an ordinal extension of $H$ in $\mathscr{C}(G)$ (see $\S 2$ ). The notion of a lex-extension was first introduced in Conrad [8] and appears in its present form in Conrad [11].

The cardinal sum $\boxplus\left\{G_{i}: i \in I\right\}$ of a family $\left\{G_{i}: i \in I\right\}$ of $l$-groups is the direct sum of this family endowed with the component-wise order.

Conrad calls an $l$-group $G$ a (finite) lexico-sum of a family $\left\{G_{1}, G_{2}, \ldots, G_{n}\right\}$ of $l$-groups provided there exists a chain $\boldsymbol{L}_{0} \subset \boldsymbol{L}_{1} \subset \cdots \subset \boldsymbol{L}_{m}=G$ of $l$-ideals of $G$ such that $\boldsymbol{L}_{k}=\boxplus \mathbf{B}(k)$ for $0 \leq k \leq m$, where

(1) $\mathbf{B}(0)=\left\{G_{1}, G_{2}, \ldots, G_{n}\right\}$;

(2) $\mathbf{B}(k+1)=\left\{G_{1}^{k+1}, G_{2}^{k+1}, \ldots, G_{j}^{k+1}\right\}$, where each $G_{i}^{k+1}$ is either a member of $\mathbf{B}(k)$ or else a proper lex-extension of the cardinal sum of two or more members of $\boldsymbol{L}_{k}$.

In Conrad [8], it is proven that an $l$-group $G$ is a finite lexico-sum of $n o$ groups if and only if $G$ contains $n$ pairwise orthogonal elements but not $n+1$ such elements (Conrad [8, Theorem 1]; see also Conrad [12, p. 2.47]). This result has come to be known as the Finite Basis Theorem; our Theorem 5.10 is a purely lattice-theoretic analog of this result and first appears in Snodgrass and Tsinakis [27]. 
In Conrad [9], the notion of a finite lexico-sum is extended to include the possibility that $\mathbf{B}(0)$ (and hence each $\mathbf{B}(k)$ ) may be infinite and $G$ is the union $\bigcup\left\{\boldsymbol{L}_{k}: k<\omega\right\}$. This extended construction is called a small lexico-sum in Conrad [9] and a restricted $\omega$ lex-sum in Conrad [11, 12]. Conrad proves that an $l$-group $G$ is a small lexico-sum of a family of $o$-groups if and only if $G$ satisfies $\mathscr{F}$ : every positive element of $G$ exceeds at most a finite number of pairwise orthogonal elements (Conrad [9, Theorem 6.1]). Our Theorem 5.8 is a purely lattice-theoretic analog of this result and includes it as a special case. In Bigard et al. [5], the term "orthofinite" is used to describe Property $\mathscr{F}$.

Our definition of an ordinal decomposition (Definition 4.1) is based upon the concept of a $\tau$ lexico-sum of multilattice groups appearing in McAlister [22]. McAlister's definition carries Conrad's ordinal notion of a lexico-sum into the more general context of multilattice groups and includes the possibility that the multilattice group $G$ is the union $\bigcup\left\{L_{\beta}: 0 \leq \beta<\tau\right\}$, where $\tau$ is an arbitrary ordinal. Conrad's definition of a lex-sum in Conrad [11] is a variation of McAlister's concept of a $\tau$ lexico-sum. The following conditions are equivalent for an $l$-group $G$ :

(1) $G$ is a lex-sum of o-groups.

(2) $G$ is finite-valued and $\mathbf{M}(G)$ satisfies $D C C$.

(3) Each filet chain in $G$ is finite.

In this setting, $\mathbf{M}(G)$ denotes the set of all maximal lex-subgroups of $G$. These are the maximal ordinal elements of the lattice $\mathscr{C}(G)$. The equivalence of conditions (2) and (3) is due to McAlister [22, Theorem 3.1], the equivalence of conditions (1) and (2) to Conrad [11, Theorem 5.2]. Our Theorem 4.11 generalizes this result.

Jaffard [18] considers the Glivenko quotient of the lattice of principal convex $l$-subgroups of an $l$-group, referring to the elements of this quotient as filets. Our Lemmas 3.1, 3.6, and 3.7 are motivated by similar results appearing in this paper.

Our definition of a filet configuration is based upon the concept of a filetchain. A hint of the connection between filet chains and lex-sums appears in Conrad's proof of Theorem 6.1 (Conrad [9]), though it is in McAlister [22] that the term "filet-chain" is introduced and a connection between these posets and lex-sums is explicitly stated. A special case of our Theorem 3.3 for l-groups appears in Conrad [11, 12] (see in particular Conrad [12, Theorem 2.22]). The equivalence of the conditions of Theorem 3.3 and the statement that a member of the class $\mathscr{I R N}$ is bialgebraic first appears in Snodgrass and Tsinakis [26].

Our definition of the set $\mathbf{C M}(\boldsymbol{L})$, Lemmas 4.2, 4.3, 4.6, and 4.8, and Construction 4.7 are adaptations of, or are motivated by, similar results appearing in Conrad [11] and Conrad [12, Chapter 3]. The characterization of $\omega$ decompositions given in Theorem 5.4 is based upon a similar theorem for $l$ groups due to Norman Reilly (see Conrad [11] or [12, p. 3.21]). A special case of Lemma 5.6 for l-groups appears in Conrad [12, p. 3.19].

\section{REFERENCES}

1. M. Anderson, P. Conrad, and J. Martinez, The lattice of convex l-subgroups of a lattice-ordered group, Lattice-Ordered Groups, Advances and Techniques (A. M. W. Glass and W. C. Holland, eds.), Kluwer Academic, 1989, pp. 105-127. 
2. M. Anderson and T. Feil, Lattice-ordered groups, an introduction, Reidel, Dordrecht, 1988.

3. R. Balbes and P. Dwinger, Distributive lattices, Univ. Missouri Press, Columbia, MO, 1974.

4. R. Beazer, Hierarchies of distributive lattices satisfying annihilator conditions, J. London Math. Soc. (2) 11 (1975), 216-222.

5. A. Bigard, K. Keimel, and S. Wolfenstein, Groupes et anneaux réticulés, Springer-Verlag, Berlin, 1977.

6. G. Bordalo, Strongly n-normal lattices, Indag. Math. (N.S.) 46 (1984), 113-125.

7. G. Bordalo and H. Priestly, Relative Ockham lattices: their order-theoretic and algebraic characterization, Glasgow Math. J. 32 (1990), 47-66.

8. P. Conrad, The structure of a lattice-ordered group with a finite number of disjoint elements, Michigan Math. J. 7 (1960), 171-180.

9. (1961), 212-240.

10. The lattice of all convex l-subgroups of a lattice-ordered group, Czechoslavak. Math. J. 15 (1965), 101-123.

11. _ Lex-subgroups of lattice-ordered groups, Czechoslavak. Math. J. 18 (1968), 86-103.

12. __ Lattice-ordered groups, Tulane Univ. lecture notes, New Orleans, 1970.

13. W. H. Cornish, Normal lattices, J. Austral. Math. Soc. 14 (1972), 200-215.

14. _ n-normal lattices, Proc. Amer. Math. Soc. 45 (1974), 48-53.

15. P. Crawley and R. P. Dilworth, Algebraic theory of lattices, Prentice-Hall, Englewoods Cliffs, NJ, 1973.

16. B. Davey, Some annihilator conditions on distributive lattices, Algebra Universalis 4 (1974), 316-322.

17. G. Grätzer, General lattice theory, Academic Press, New York, 1978.

18. P. Jaffard, Contribution a l'etude des groupes ordonees, J. Math. Pures Appl. 32 (1953), 208-280.

19. P. Johnstone, Stone spaces, Cambridge Stud. Adv. Math., vol. 3, Cambridge Univ. Press, London and New York, 1980.

20. M. Mandelker, Relative annihilators in lattices, Duke Math. J. 37 (1970), 377-386.

21. G. Markowsky and B. K. Rosen, Bases for chain-complete posets, IBM J. Res. Develop. 20 (1976), 138-147.

22. D. B. McAlister, On multilattice groups. II, Proc. Cambridge Philos. Soc. (1966), 149-164.

23. R. McKenzie, G. McNulty, and W. Taylor, Algebras, lattices, varieties, vol. 1, Wadsworth and Brooks/Cole, Monterey, CA, 1987.

24. A. Monteiro, L'arithmetique des filtres et les espaces topologiques, De Segundo Symposium de Matematicas-Villavicencio (Mendoza, Buenos Aires), 1954, pp. 129-162.

25. __ L'arithmetique des filtres et les espaces topologiques. I, II, Notas Lógica Mat. (1974), 29-30.

26. J. Snodgrass and C. Tsinakis, Finite-valued algebraic lattices, Algebra Universalis 30 (1993), 311-318.

27.

28. A. Zaanen, Riesz spaces. II, North-Holland Math. Library, vol. 30, North-Holland, Amsterdam, 1983.

Department of Mathematics, Vanderbilt University, Nashville, Tennessee 37235

E-mail address, Constantine Tsinakis: tsinakis@athena.cas.vanderbilt.edu 